\title{
Axisymmetric Large Deflection Elastic Analysis of Hollow Annular Membranes under Transverse Uniform Loading
}

\author{
Jun-Yi Sun ${ }^{1,2, *(\mathbb{D},}$ Qi Zhang ${ }^{1}, \mathrm{Xue} \mathrm{Li}^{1}$ and Xiao-Ting $\mathrm{He}^{1,2} \mathbb{D}$ \\ 1 School of Civil Engineering, Chongqing University, Chongqing 400045, China; \\ 202016021045@cqu.edu.cn (Q.Z.); 20161602025t@cqu.edu.cn (X.L.); hexiaoting@cqu.edu.cn (X.-T.H.) \\ 2 Key Laboratory of New Technology for Construction of Cities in Mountain Area (Chongqing University), \\ Ministry of Education, Chongqing 400045, China \\ * Correspondence: sunjunyi@cqu.edu.cn; Tel.: +86-(0)23-65120720
}

check for updates

Citation: Sun, J.-Y.; Zhang, Q.; Li, X.; $\mathrm{He}, \mathrm{X} .-\mathrm{T}$. Axisymmetric Large Deflection Elastic Analysis of Hollow Annular Membranes under Transverse Uniform Loading. Symmetry 2021, 13, 1770. https:/ / doi.org/10.3390/sym13101770

Academic Editor: Massimo Latour

Received: 3 September 2021

Accepted: 20 September 2021

Published: 23 September 2021

Publisher's Note: MDPI stays neutral with regard to jurisdictional claims in published maps and institutional affiliations.

Copyright: (c) 2021 by the authors. Licensee MDPI, Basel, Switzerland. This article is an open access article distributed under the terms and conditions of the Creative Commons Attribution (CC BY) license (https:/ / creativecommons.org/licenses/by/ $4.0 /)$.

\begin{abstract}
The anticipated use of a hollow linearly elastic annular membrane for designing elastic shells has provided an impetus for this paper to investigate the large deflection geometrically nonlinear phenomena of such a hollow linearly elastic annular membrane under transverse uniform loads. The so-called hollow annular membranes differ from the traditional annular membranes available in the literature only in that the former has the inner edge attached to a movable but weightless rigid concentric circular ring while the latter has the inner edge attached to a movable but weightless rigid concentric circular plate. The hollow annular membranes remove the transverse uniform loads distributed on "circular plate" due to the use of "circular ring" and result in a reduction in elastic response. In this paper, the large deflection geometrically nonlinear problem of an initially flat, peripherally fixed, linearly elastic, transversely uniformly loaded hollow annular membrane is formulated, the problem formulated is solved by using power series method, and its closed-form solution is presented for the first time. The convergence and effectiveness of the closed-form solution presented are investigated numerically. A comparison between closed-form solutions for hollow and traditional annular membranes under the same conditions is conducted, to reveal the difference in elastic response, as well as the influence of different closed-form solutions on the anticipated use for designing elastic shells.
\end{abstract}

Keywords: annular membrane; uniform transverse loading; structural analysis; large deflection; power series method; closed-form solution

\section{Introduction}

The so-called circular or annular membrane problem is well known, that is, the problem of axisymmetric deformation and deflection of an initially flat circular or annular membrane subjected to uniform transverse (lateral) or normal loading. In the existing literature, however, attention is mainly focused on the circular membrane problem, while the closed-form solutions for the annular membrane problem are available in a few cases.

H. Hencky was the first scholar to deal with analytically the circular membrane problem, who used the power series method to solve the system of equations of large deflection of thin plates with vanishing bending stiffness [1]. This system of equations was, originally by A. Föppl, derived from the classical Föppl-von Kármán equations of large deflection of thin plates [2-4]. Therefore, the circular membrane problem is also called Föppl-Hencky membrane problem or simply Hencky problem, but H. Hencky originally dealt with only the case of a stress-free circular membrane subjected to uniform transverse loading [1]. A computational error in the power series solution which was presented by H. Hencky in 1915 [1] was subsequently corrected by Chien in 1948 [5] and Alekseev in 1953 [6], respectively. This solution is usually called Hencky solution, which is often cited in some studies of related issues [7-21]. The closed-form solutions of the circular membrane problem under normal loading are available in a few cases in the 
existing literature [22-24]. Fichter [22] dealt analytically with the problem of axisymmetric deformation and deflection of a stress-free circular membrane under gas pressure (uniform normal loading), where the horizontal component of the applied gas pressure was included. This horizontal component is an extra component in comparison with the Hencky solution and was neglected in Campbell's solution [23]. Shi et al. [24] presented the closed-form solution for the problem of axisymmetric deformation and deflection of a prestressed circular membrane under gas pressure.

S. A. Alekseev was the first scholar to deal with analytically the annular membrane problem, who considered an annular membrane structure containing a movable rigid concentric circular thin plate on which a transverse concentrated force acts, i.e., an annular membrane with an immovable and indeformable outer edge and a movable but indeformable inner edge connected to a movable but weightless rigid concentric circular thin plate loaded by a transverse concentrated force at its center [25]. The problem which was originally dealt with by Alekseev [25] is actually the problem of axisymmetric deformation and deflection of an initially flat, peripherally fixed annular membrane subjected to the transverse loads distributed linearly uniformly along its movable but indeformable inner edge, but Alekseev [25] failed to give a complete solution to the problem he was considering. The closed-form solution presented by Alekseev [25] is valid only when the Poisson's ratio of membrane is less than 1/3. Sun et al. [26] presented the complete closed-form solution of the Alekseev's problem, which is suitable for the case where the Poisson's ratio of membrane may be less or greater than 1/3, or equal to 1/3. Yang et al. [27] extended the closed-form solution presented by Sun et al. [26] to include the case of an annular membrane with pre-stress. Lian et al. [28] dealt with analytically the problem of axisymmetric deformation and deflection of the classic Alekseev's annular membrane structure subjected to uniform transverse loading, where both the entire annular membrane and the movable rigid concentric circular thin plate are subjected to uniformly distributed transverse loads. The closed-form solution presented by Lian et al. was incorporated into improvement of a capacitive pressure sensor [28].

In this paper, attention is focused on the problem of axisymmetric deformation and deflection of a hollow annular membrane structure subjected to uniform transverse loading, an initially flat, linearly elastic, transversely uniformly loaded annular membrane with an immovable and indeformable outer edge and a movable but indeformable inner edge attached to a movable but weightless rigid concentric ring. The anticipated use of the closed-form solution of this problem for elastic shell design has provided an impetus for us to investigate this large deflection phenomenon. We are carrying out a research on what is called an elastic conjugate shell of revolution, which was funded by the National Natural Science Foundation of China (Grant No. 11772072). The so-called elastic conjugate shells of revolution are considered to have an optimal relationship between structural deformation and structural stress state and thus have a strong stable bearing capacity. The closed-form solution of this problem can be used to design such an elastic conjugate shell of revolution, a hollow annular conjugate shell of revolution that bears uniformly distributed transverse loads.

Essential to the design of such a hollow annular conjugate shell of revolution is the ability to obtain the shape and stress distribution of the hollow annular membrane structure under uniformly distributed transverse loads. Therefore, the main task of this paper is to give the closed-form solutions of deflection and stress of the problem under consideration, which are not yet available in the existing literature. In the following section, the problem of axisymmetric deformation and deflection of the hollow annular membrane structure subjected to uniform transverse loading is formulated and is, by using power series method, solved. In Section 3, some important issues are discussed, such as the convergence and effectiveness of the closed-form solution presented, and the difference between the closedform solutions presented in this paper and in Lian et al. [28] which are both suitable for uniform transverse loading. Concluding remarks are given in Section 4. 


\section{Membrane Equations and Solutions}

Let us consider a rotationally symmetric, linearly elastic, initially flat annular membrane with Poisson's ratio $v$, Young's modulus of elasticity $E$, inner radius $b$, outer radius $a$, and thickness $h$, whose outer edge is tightly fixed to an immovable clamping device and inner edge is tightly clamped by a movable but weightless rigid concentric circular ring, resulting in an immovable and indeformable outer edge and a movable but indeformable inner edge attached to a movable but weightless rigid concentric circular ring. A uniformly distributed transverse load $q$ is quasi-statically applied onto the annular membrane, resulting in an out-of-plane displacement (deflection) of the annular membrane, as shown in Figure 1, where a cylindrical coordinate system $(r, \varphi, w)$ is introduced, whose coordinate origin is placed in the centroid of the geometric intermediate plane of the initially flat annular membrane and its polar coordinate plane $(r, \varphi)$ is located in the plane in which the geometric intermediate plane is located, $o$ denotes the coordinate origin of the coordinate system $(r, \varphi, w), r$ denotes the radial coordinate, $\varphi$ denotes the angle coordinate (but it is not represented in Figure 1), and $w$ denotes the axial coordinate whose positive direction corresponds to the deflecting direction of the annular membrane.

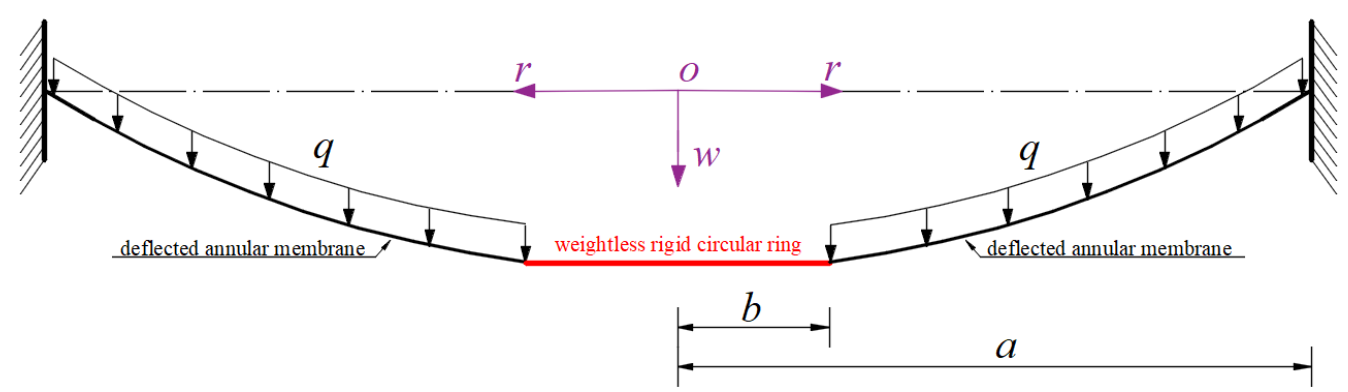

Figure 1. Sketch of the hollow annular membrane under uniformly distributed transverse loads $q$, where the dash-dot line represents the geometric intermediate plane of the initially flat hollow annular membrane and there is no load $q$ in the hollow area $(0 \leq r \leq b)$.

A free body of a piece of annular membrane with radius $r(b \leq r \leq a)$ is removed from the central portion of the deflected hollow annular membrane, to study the static problem of equilibrium of this free body under the joint action of the external active force $\left(\pi r^{2}-\pi b^{2}\right) q$ produced by the transverse loads $q$ and the reactive force $2 \pi r \sigma_{r} h$ produced by the membrane force $\sigma_{r} h$ acted on the boundary $r$, as shown in Figure 2, where $\sigma_{r}$ denotes the radial stress and $\theta$ denotes the slope angle of the deflected annular membrane.

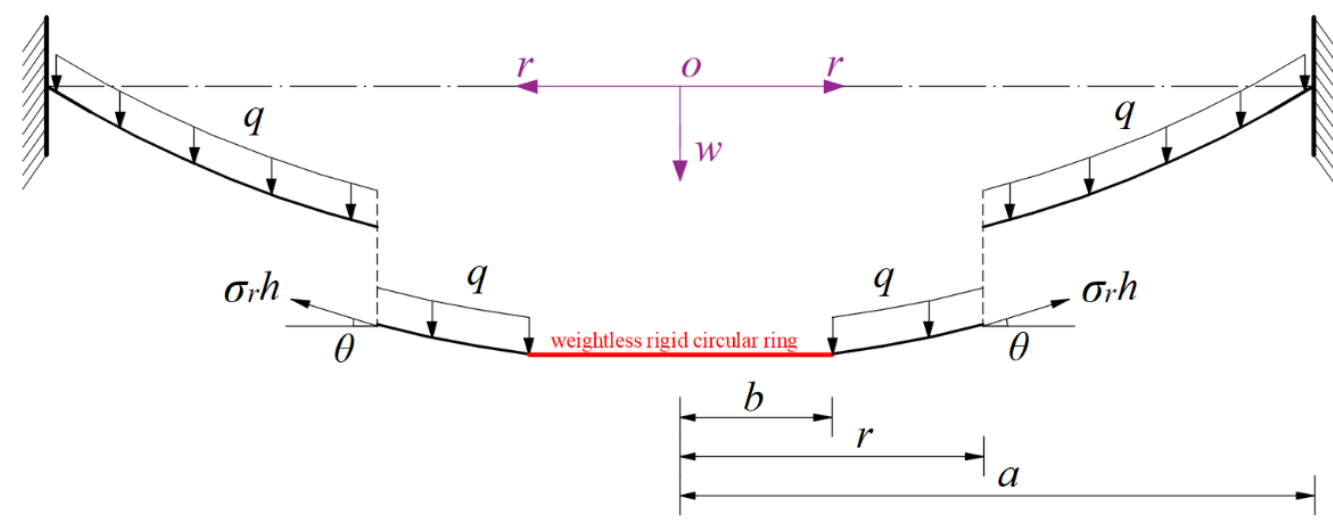

Figure 2. A free body of radius $b \leq r \leq a$ removed from the deflected hollow annular membrane.

Therefore, the so-called out-of-plane equilibrium equation can be obtained from the equilibrium condition that the resultant force in the transverse (vertical) direction is equal to zero, that is

$$
2 \pi r \sigma_{r} h \sin \theta=\left(\pi r^{2}-\pi b^{2}\right) q .
$$


If the transverse displacement at $r$ is denoted by $w(r)$, then

$$
\sin \theta \approx \tan \theta=-\frac{\mathrm{d} w}{\mathrm{~d} r} .
$$

Substituting Equation (2) into Equation (1), the out-of-plane equilibrium equation can be written as

$$
2 r \sigma_{r} h\left(-\frac{\mathrm{d} w}{\mathrm{~d} r}\right)=\left(r^{2}-b^{2}\right) q .
$$

In the horizontal direction parallel to the initially flat annular membrane, there are the actions of the radial membrane force $\sigma_{r} h$ and the circumferential membrane force $\sigma_{t} h$, where $\sigma_{t}$ denotes circumferential stress. Therefore, the so-called in-plane equilibrium equation may be written as [26]

$$
\frac{\mathrm{d}}{\mathrm{d} r}\left(r \sigma_{r} h\right)-\sigma_{t} h=0 .
$$

If the radial strain, circumferential strain and radial displacement are denoted by $e_{r}, e_{t}$, and $u(r)$, respectively, then the relationship between displacement and strain, the usually so-called geometric equations, may be written as

$$
e_{r}=\frac{\mathrm{d} u}{\mathrm{~d} r}+\frac{1}{2}\left(\frac{\mathrm{d} w}{\mathrm{~d} r}\right)^{2}
$$

and

$$
e_{t}=\frac{u}{r}
$$

Equation (5) is a second-order geometrically nonlinear equation. Moreover, the relationship between strain and stress, the so-called physical equations, is still assumed to satisfy linear elasticity

$$
\sigma_{r}=\frac{E}{1-v^{2}}\left(e_{r}+v e_{t}\right)
$$

and

$$
\sigma_{t}=\frac{E}{1-v^{2}}\left(e_{t}+v e_{r}\right) .
$$

Substituting Equations (5) and (6) into Equations (7) and (8) yields

$$
\sigma_{r}=\frac{E}{1-v^{2}}\left[\frac{\mathrm{d} u}{\mathrm{~d} r}+\frac{1}{2}\left(\frac{\mathrm{d} w}{\mathrm{~d} r}\right)^{2}+v \frac{u}{r}\right]
$$

and

$$
\sigma_{t}=\frac{E}{1-v^{2}}\left[\frac{u}{r}+v \frac{\mathrm{d} u}{\mathrm{~d} r}+\frac{v}{2}\left(\frac{\mathrm{d} w}{\mathrm{~d} r}\right)^{2}\right] .
$$

Eliminating $\mathrm{d} w / \mathrm{d} x$ from Equations (9) and (10) and further using Equation (4) yield

$$
\frac{u}{r}=\frac{1}{E h}\left(\sigma_{t} h-v \sigma_{r} h\right)=\frac{1}{E h}\left[\frac{\mathrm{d}}{\mathrm{d} r}\left(r \sigma_{r} h\right)-v \sigma_{r} h\right] .
$$

After substituting the $u$ of Equation (11) into Equation (9), then the usually so-called consistency equation can be written as

$$
r \frac{\mathrm{d}}{\mathrm{d} r}\left[\frac{1}{r} \frac{\mathrm{d}}{\mathrm{d} r}\left(r^{2} \sigma_{r}\right)\right]+\frac{E}{2}\left(\frac{\mathrm{d} w}{\mathrm{~d} r}\right)^{2}=0 .
$$

Equations (3), (4) and (12) are three equations for the solutions of $\sigma_{r}, \sigma_{t}$ and $w$. 
Due to the movable but indeformable inner edge at $r=b$ and the immovable and indeformable outer edge at $r=a$, the boundary conditions for solving Equations (3), (4) and (12) are

$$
\begin{aligned}
& e_{t}=\frac{u}{r}=0 \text { at } r=b, \\
& e_{t}=\frac{u}{r}=0 \text { at } r=a
\end{aligned}
$$

and

$$
w=0 \text { at } r=a .
$$

Let us introduce the following nondimensional variables

$$
Q=\frac{a q}{E h}, W=\frac{w}{a}, S_{r}=\frac{\sigma_{r}}{E}, S_{t}=\frac{\sigma_{t}}{E}, \alpha=\frac{b}{a}, x=\frac{r}{a},
$$

and transform Equations (3), (4), (11)-(15) into

$$
\begin{gathered}
2 x S_{r}\left(-\frac{\mathrm{d} W}{\mathrm{~d} x}\right)=\left(x^{2}-\alpha^{2}\right) Q, \\
\frac{\mathrm{d}\left(x S_{r}\right)}{\mathrm{d} x}-S_{t}=0, \\
\frac{u}{r}=(1-v) S_{r}+x \frac{\mathrm{d} S_{r}}{\mathrm{~d} x}, \\
x^{2} \frac{\mathrm{d}^{2} S_{r}}{\mathrm{~d} x^{2}}+3 x \frac{\mathrm{d} S_{r}}{\mathrm{~d} x}+\frac{1}{2}\left(\frac{\mathrm{d} W}{\mathrm{~d} x}\right)^{2}=0, \\
(1-v) S_{r}+x \frac{\mathrm{d} S_{r}}{\mathrm{~d} x}=0 \text { at } x=\alpha, \\
(1-v) S_{r}+x \frac{\mathrm{d} S_{r}}{\mathrm{~d} x}=0 \text { at } x=1
\end{gathered}
$$

and

$$
W=0 \text { at } x=1 .
$$

Eliminating $\mathrm{d} W / \mathrm{d} x$ from Equations (17) and (20), we can obtain an equation which contains only $S_{r}$

$$
x^{2} \frac{\mathrm{d}^{2} S_{r}}{\mathrm{~d} x^{2}}+3 x \frac{\mathrm{d} S_{r}}{\mathrm{~d} x}+\frac{\left(x^{2}-\alpha^{2}\right)^{2} Q^{2}}{8 x^{2} S_{r}^{2}}=0 .
$$

In view of the physical phenomenon that the values of stress, strain and displacement are finite within $\alpha \leq x \leq 1$, we can expand $S_{r}$ and $W$ into the power series of the $x-\beta$, i.e., letting

$$
S_{r}=\sum_{i=0}^{\infty} c_{i}(x-\beta)^{i}
$$

and

$$
W=\sum_{i=0}^{\infty} d_{i}(x-\beta)^{i},
$$

where $\beta=(1+\alpha) / 2$. For convenience we introduce $X=x-\beta$, then Equations (17), (24)-(26) can be transformed into

$$
\begin{gathered}
2(X+\beta) S_{r}\left(-\frac{\mathrm{d} W}{\mathrm{~d} X}\right)=\left[(X+\beta)^{2}-\alpha^{2}\right] Q \\
(X+\beta)^{2} \frac{\mathrm{d}^{2} S_{r}}{\mathrm{~d} X^{2}}+3(X+\beta) \frac{\mathrm{d} S_{r}}{\mathrm{~d} X}+\frac{\left[(X+\beta)^{2}-\alpha^{2}\right]^{2} Q^{2}}{8(X+\beta)^{2} S_{r}^{2}}=0,
\end{gathered}
$$




$$
S_{r}=\sum_{i=0}^{\infty} c_{i} X^{i}
$$

and

$$
W=\sum_{i=0}^{\infty} d_{i} X^{i}
$$

Substituting Equation (29) into Equation (28) and letting the sums of all coefficients of the same powers of the $X$ be equal to zero yield a system of equations for determining the recursion for the coefficients $c_{i}$. The solution to this system of equations shows that the coefficients $c_{i}(i=2,3,4, \ldots)$ can be expressed into the polynomial functions with regard to the first two coefficients $c_{0}$ and $c_{1}$ (see Appendix A). Further, by substituting Equation (29) (where $c_{i}$ should be expressed by $c_{0}$ and $c_{1}$, see Appendix A) and Equation (30) into Equation (27), the coefficients $d_{i}(i=1,2,3, \ldots)$ can also be expressed into $c_{0}$ and $c_{1}$ (see Appendix B).

The remaining three coefficients $d_{0}, c_{0}$ and $c_{1}$ are the so-called undetermined constants, which depend on the specific problem addressed and can be determined by using the boundary conditions Equations (21)-(23) as follows. Substituting Equation (25) (where $c_{i}$ should be expressed by $c_{0}$ and $c_{1}$, see Appendix A) into the boundary conditions Equations (21) and (22) yield

$$
(1-v) \sum_{i=0}^{\infty} c_{i}(\alpha-\beta)^{i}+\alpha \sum_{i=1}^{\infty} i c_{i}(\alpha-\beta)^{i-1}=0
$$

and

$$
(1-v) \sum_{i=0}^{\infty} c_{i}(1-\beta)^{i}+\sum_{i=1}^{\infty} i c_{i}(1-\beta)^{i-1}=0 .
$$

Obviously, Equations (31) and (32) contain only $c_{0}$ and $c_{1}$. Therefore, the values of $c_{0}$ and $c_{1}$ can be determined by simultaneously solving Equations (31) and (32), and the expression of $S_{r}$ can thus be determined. Further, substituting Equation (26) (where $d_{i}$ should be expressed by $c_{0}$ and $c_{1}$, see Appendix B) into the boundary condition Equation (23) yields

$$
d_{0}=-\sum_{i=1}^{\infty} d_{i}(1-\beta)^{i}
$$

Therefore, with the known $c_{0}$ and $c_{1}$ the value of $d_{0}$ can be determined by Equation (33), and the expression of $W$ can also be determined. As for the expression of $S_{t}$, with the known expression of $S_{t}$, it can easily be determined by Equation (18).

The problem under consideration is thus solved analytically, and its closed-form solutions of stress and deflection are given.

\section{Results and Discussion}

Let us first discuss the convergence of the closed-form solution obtained in Section 2. It may be seen, from the derivation in Section 2, that the closed-form solutions of $\mathrm{S}_{r}$ and $W$ are obtained by using the power series method and the power series coefficients $c_{i}(i=2,3,4, \ldots)$ and $d_{i}(i=1,2,3, \ldots)$ are expressed into the polynomial in the undetermined constants $c_{0}$ and $c_{1}$. Therefore, due to these somewhat intractable expressions of the coefficients $b_{i}$ and $c_{i}$ (see Appendices A and B), we have to prove the convergence of the special solutions of stress and deflection, rather than that of their general solutions. To this end, we consider a numerical example of an annular membrane with thickness $h=0.2 \mathrm{~mm}$, inner radius $b=10 \mathrm{~mm}$, outer radius $a=20 \mathrm{~mm}$, Young's modulus of elasticity $E=7.84 \mathrm{MPa}$ and Poisson's ratio $v=0.47$ under the action of the uniformly distributed transverse loads $q=0.0003 \mathrm{MPa}$. Substituting the values of $a, b, h, E, v, q$ into Equation (16) yields $Q=0.00382653$ and $\alpha=1 / 2(\beta=(1+\alpha) / 2=3 / 4)$. 
For convenience of operation, the infinite power series in Equations (31)-(33) have to be truncated to $n$ terms, that is

$$
\begin{aligned}
(1-v) \sum_{i=0}^{n} c_{i}(\alpha-\beta)^{i}+\alpha \sum_{i=1}^{n} i c_{i}(\alpha-\beta)^{i-1} & =0, \\
(1-v) \sum_{i=0}^{n} c_{i}(1-\beta)^{i}+\sum_{i=1}^{n} i c_{i}(1-\beta)^{i-1} & =0
\end{aligned}
$$

and

$$
d_{0}=-\sum_{i=1}^{n} d_{i}(1-\beta)^{i}
$$

For a given value of the parameter $n$ in Equations (34)-(36), Equations (34) and (35) are used to determine the specific numerical values of the undetermined constants $c_{0}$ and $c_{1}$, and with the known $c_{0}$ and $c_{1}$ the value of $d_{0}$ can further be determined by Equation (36). We start calculating the numerical values of the undetermined constants $c_{0}, c_{1}$ and $d_{0}$ from $n=2$. The results of numerical value calculation of the undetermined constants $c_{0}, c_{1}$ and $d_{0}$ are listed in Table 1 , and the variation of $c_{0}, c_{1}$ and $d_{0}$ with $n$ are shown in Figures 3-5.

Table 1. The results of numerical value calculation of $c_{0}, c_{1}$ and $d_{0}$.

\begin{tabular}{cccc}
\hline $\boldsymbol{n}$ & $\boldsymbol{c}_{\mathbf{0}}$ & $\boldsymbol{c}_{\mathbf{1}}$ & $\boldsymbol{d}_{\mathbf{0}}$ \\
\hline 2 & 0.00684094 & -0.00615869 & 0.04503607 \\
3 & 0.01084290 & -0.00559109 & 0.02746185 \\
4 & 0.00811219 & -0.00401906 & 0.03697619 \\
5 & 0.00861287 & -0.00392109 & 0.03464763 \\
6 & 0.00832182 & -0.00376782 & 0.03588948 \\
7 & 0.00839685 & -0.00375182 & 0.03553708 \\
8 & 0.00835166 & -0.00372857 & 0.03573562 \\
9 & 0.00836447 & -0.00372574 & 0.03567455 \\
10 & 0.00835660 & -0.00372169 & 0.03570937 \\
11 & 0.00835878 & -0.00372120 & 0.03569895 \\
12 & 0.00835750 & -0.00372054 & 0.03570462 \\
13 & 0.00835783 & -0.00372046 & 0.03570301 \\
15 & 0.00835764 & -0.00372036 & 0.03570385 \\
\hline
\end{tabular}

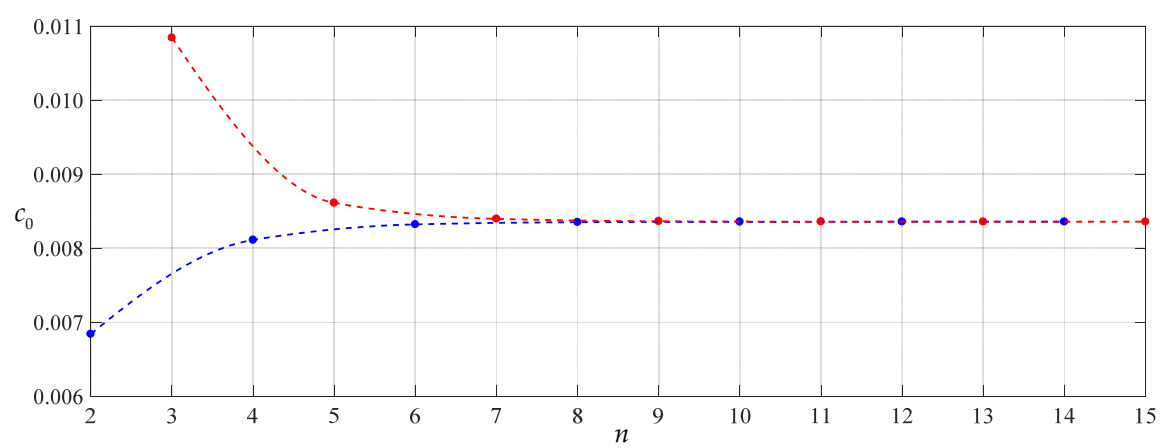

Figure 3. Variation of $c_{0}$ with $n$, where the blue refers to the case when $n$ takes even and the red refers to the case when $n$ takes odd. 


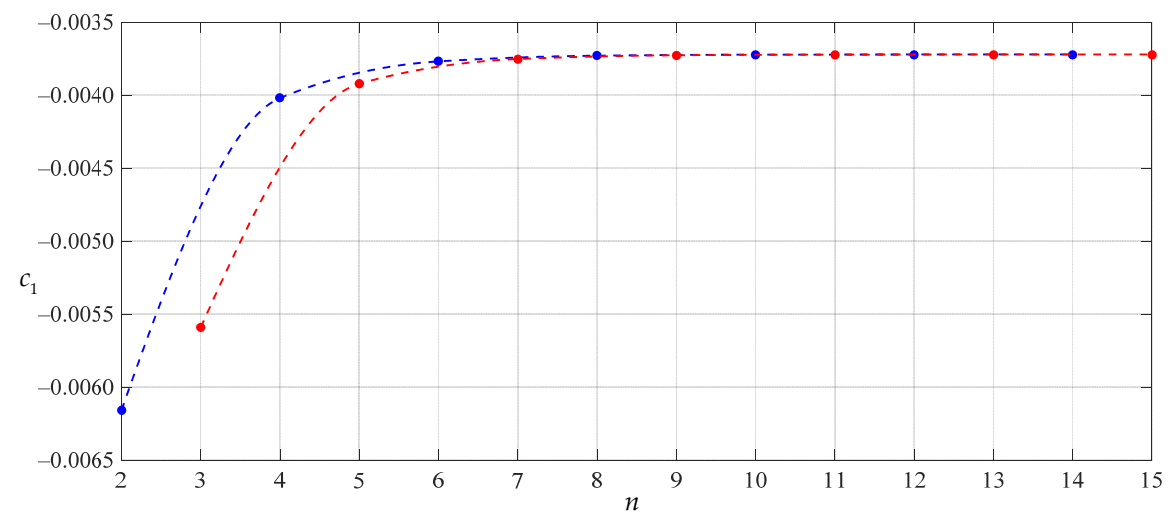

Figure 4. Variation of $c_{1}$ with $n$, where the blue refers to the case when $n$ takes even and the red refers to the case when $n$ takes odd.

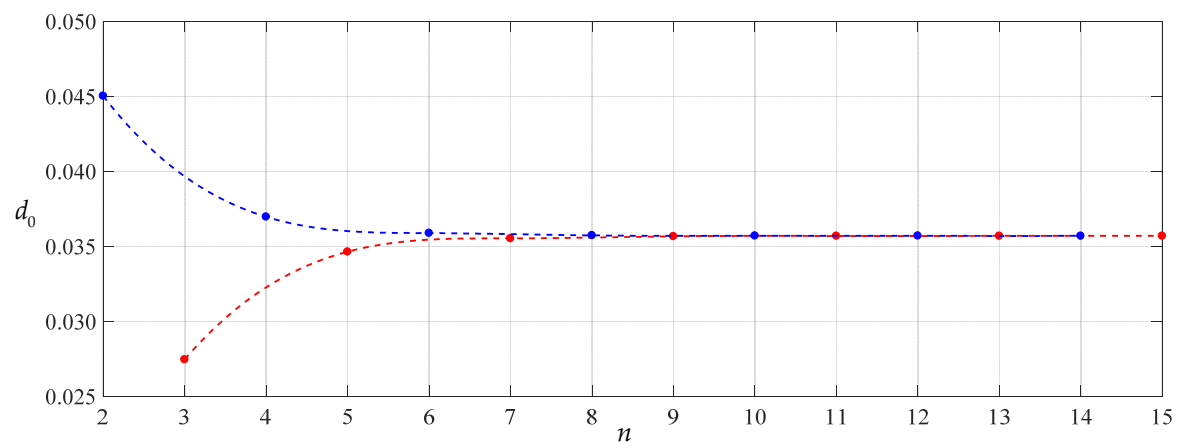

Figure 5. Variation of $d_{0}$ with $n$, where the blue refers to the case when $n$ takes even and the red refers to the case when $n$ takes odd.

It may be seen, from Figures $3-5$, that the data sequence for $c_{0}, c_{1}$ and $d_{0}$ converge rapidly to their theoretical values (their exact values) as the parameter $n$ is increased. Therefore, the undetermined constants $c_{0}, c_{1}$ and $d_{0}$ can finally take $0.00835769,-0.00372035$ and 0.03570362 , respectively (the values at $n=15$, see Table 1 ). For examining the convergence of the special solutions of stress and deflection at both ends of the closed interval of the independent variable, i.e., at $x=1$ or $r=a$ and at $x=\alpha$ or $r=b$, the numerical values of $c_{i}(1-\beta)^{i}, c_{i}(\alpha-\beta)^{i}, d_{i}(1-\beta)^{i}$ and $d_{i}(\alpha-\beta)^{i}$ are calculated by using $\alpha=1 / 2, \beta=3 / 4$ and the values of $c_{0}$ and $c_{1}$ at $n=15$, which are listed in Tables 2 and 3. The variation of $c_{i}(1-\beta)^{i}, c_{i}(\alpha-\beta)^{i}, d_{i}(1-\beta)^{i}$ and $d_{i}(\alpha-\beta)^{i}$ with $i$ are shown in Figures $6-9$, showing that the special solutions of stress and deflection converge very well.

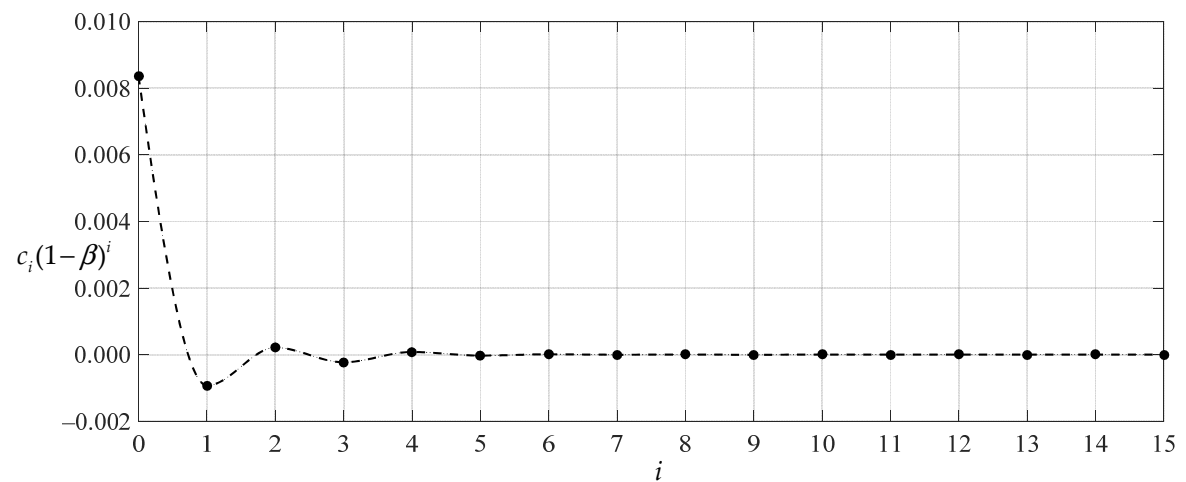

Figure 6. Variation of the $c_{i}(1-\beta)^{i}$ at $n=15$ with $i$, where $\beta=3 / 4$. 
Table 2. The calculated values of $c_{i}(1-\beta)^{i}$ and $c_{i}(\alpha-\beta)^{i}$ at $n=15$, where $\alpha=1 / 2$ and $\beta=3 / 4$.

\begin{tabular}{ccc}
\hline $\boldsymbol{i}$ & $\boldsymbol{c}_{\boldsymbol{i}} \mathbf{( 1 - \beta ) ^ { \boldsymbol { i } }}$ & $\boldsymbol{c}_{\boldsymbol{i}}(\boldsymbol{\alpha}-\boldsymbol{\beta})^{\boldsymbol{i}}$ \\
\hline 0 & 0.00835769 & 0.00835769 \\
1 & -0.00093009 & 0.00093009 \\
2 & 0.00021232 & 0.00021232 \\
3 & -0.00023105 & 0.00023105 \\
4 & $7.92290026 \times 10^{-5}$ & $7.92290026 \times 10^{-5}$ \\
5 & $-2.96216622 \times 10^{-5}$ & $2.96216622 \times 10^{-5}$ \\
6 & $9.62752906 \times 10^{-6}$ & $9.62752906 \times 10^{-6}$ \\
7 & $-3.42910780 \times 10^{-6}$ & $3.42910780 \times 10^{-6}$ \\
8 & $1.22656512 \times 10^{-6}$ & $1.22656512 \times 10^{-6}$ \\
9 & $-4.64693627 \times 10^{-7}$ & $4.64693627 \times 10^{-7}$ \\
10 & $1.74308977 \times 10^{-7}$ & $1.74308977 \times 10^{-7}$ \\
11 & $-6.52196918 \times 10^{-8}$ & $6.52196918 \times 10^{-8}$ \\
12 & $2.37687733 \times 10^{-8}$ & $2.37687733 \times 10^{-8}$ \\
13 & $-8.55038019 \times 10^{-9}$ & $8.55038019 \times 10^{-9}$ \\
14 & $3.03425389 \times 10^{-9}$ & $3.03425389 \times 10^{-9}$ \\
15 & $-1.07537902 \times 10^{-9}$ & $1.07537902 \times 10^{-9}$ \\
\hline
\end{tabular}

Table 3. The calculated values of $d_{i}(1-\beta)^{i}$ and $d_{i}(\alpha-\beta)^{i}$ at $n=15$, where $\alpha=1 / 2$ and $\beta=3 / 4$.

\begin{tabular}{ccc}
\hline $\boldsymbol{i}$ & $\boldsymbol{d}_{\boldsymbol{i}} \mathbf{( 1 - \beta ) ^ { \boldsymbol { i } }}$ & $\boldsymbol{d}_{\boldsymbol{i}}(\boldsymbol{\alpha}-\boldsymbol{\beta})^{\boldsymbol{i}}$ \\
\hline 0 & 0.03570362 & 0.03570362 \\
1 & -0.02384612 & 0.02384612 \\
2 & -0.01166018 & -0.01166018 \\
3 & $4.34075197 \times 10^{-5}$ & $-4.34075197 \times 10^{-5}$ \\
4 & $-1.89718510 \times 10^{-4}$ & $-1.89718510 \times 10^{-4}$ \\
5 & $-5.41777244 \times 10^{-5}$ & $5.41777244 \times 10^{-5}$ \\
6 & $8.46365300 \times 10^{-6}$ & $8.46365300 \times 10^{-6}$ \\
7 & $-5.52803048 \times 10^{-6}$ & $5.52803048 \times 10^{-6}$ \\
8 & $3.65886050 \times 10^{-7}$ & $3.65886050 \times 10^{-7}$ \\
9 & $-7.99963631 \times 10^{-8}$ & $7.99963631 \times 10^{-8}$ \\
10 & $-6.10745278 \times 10^{-8}$ & $-6.10745278 \times 10^{-8}$ \\
11 & $7.58270646 \times 10^{-9}$ & $-7.58270646 \times 10^{-9}$ \\
12 & $-1.53622173 \times 10^{-9}$ & $-1.53622173 \times 10^{-9}$ \\
13 & $-1.68207783 \times 10^{-9}$ & $1.68207783 \times 10^{-9}$ \\
14 & $6.15674878 \times 10^{-10}$ & $6.15674878 \times 10^{-10}$ \\
15 & $-2.18144847 \times 10^{-10}$ & $2.18144847 \times 10^{-10}$ \\
\hline
\end{tabular}

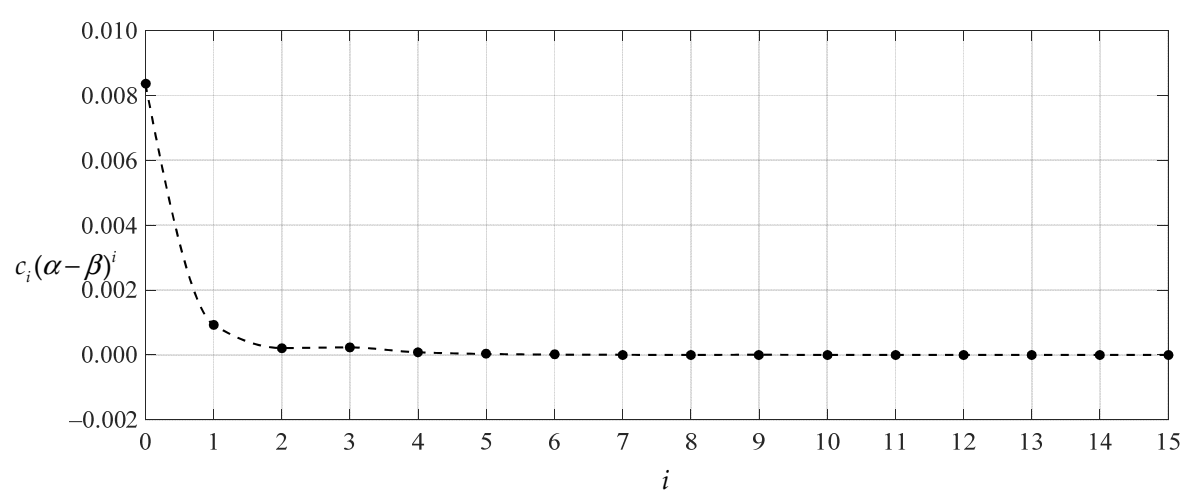

Figure 7. Variation of the $c_{i}(\alpha-\beta)^{i}$ at $n=15$ with $i$, where $\alpha=1 / 2$ and $\beta=3 / 4$. 


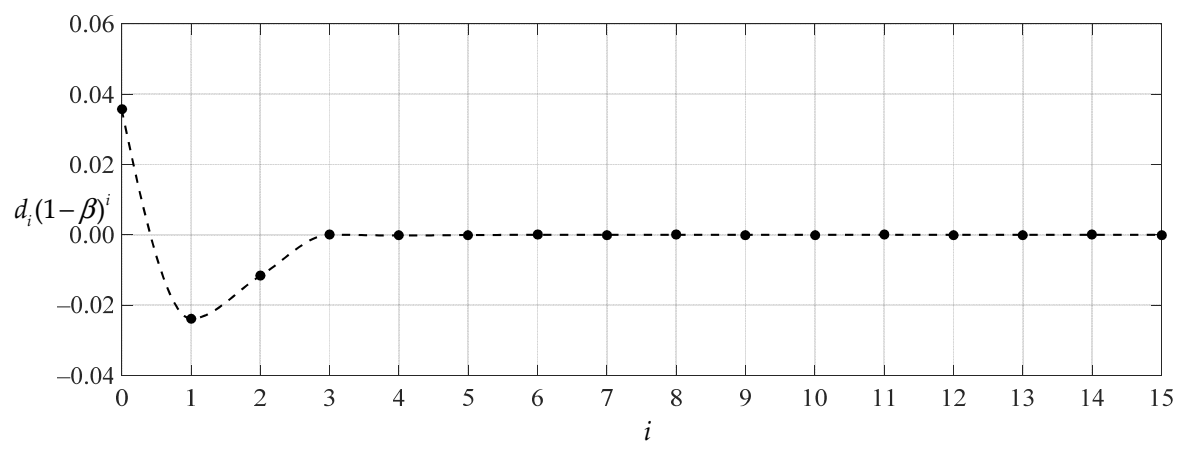

Figure 8. Variation of the $d_{i}(1-\beta)^{i}$ at $n=15$ with $i$, where $\beta=3 / 4$.

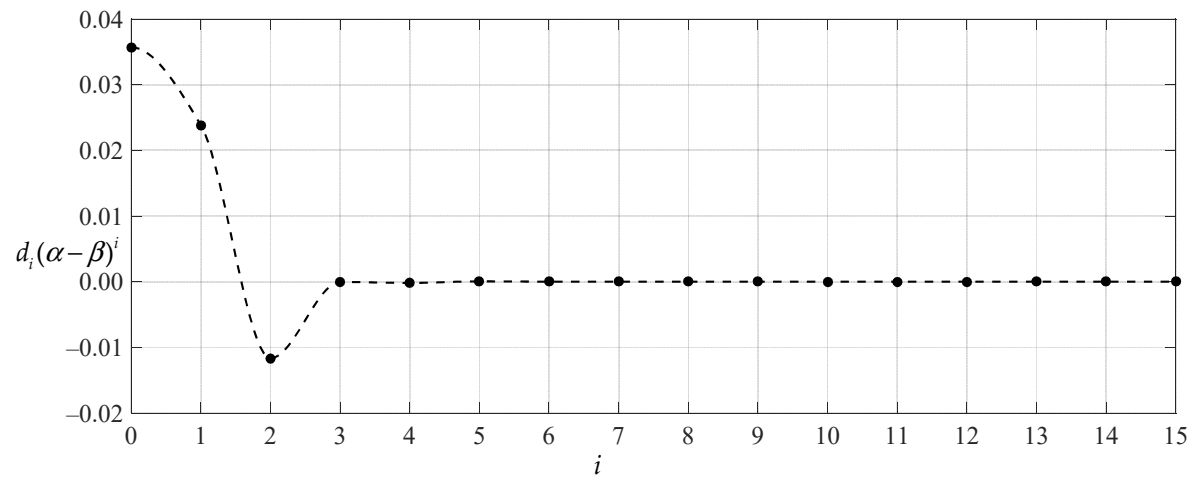

Figure 9. Variation of the $d_{i}(\alpha-\beta)^{i}$ at $n=15$ with $i$, where $\alpha=1 / 2$ and $\beta=3 / 4$.

Now, let us address the validity of the closed-form solution obtained in Section 2, which can be proved by examining whether it, under the same action of the uniformly distributed transverse loads $q=0.0003 \mathrm{MPa}$, gets closer and closer to the Hencky solution as the inner radius $b$ of the annular membrane gradually goes down. Obviously, a circular membrane can approximately be regarded as an annular membrane with an inner radius $b \rightarrow 0$. Therefore, the closed-form solution obtained in Section 2 should get closer and closer to the Hencky solution (which is for circular membrane problem) as the inner radius b of the annular membrane gradually goes to zero. Figure 10 shows the trend that the closed-form solution obtained in Section 2 approaches the Hencky solution with the decrease of the inner radius $b$ of the annular membrane, which to some extent shows that the closed-form solution obtained in Section 2 is basically reliable.

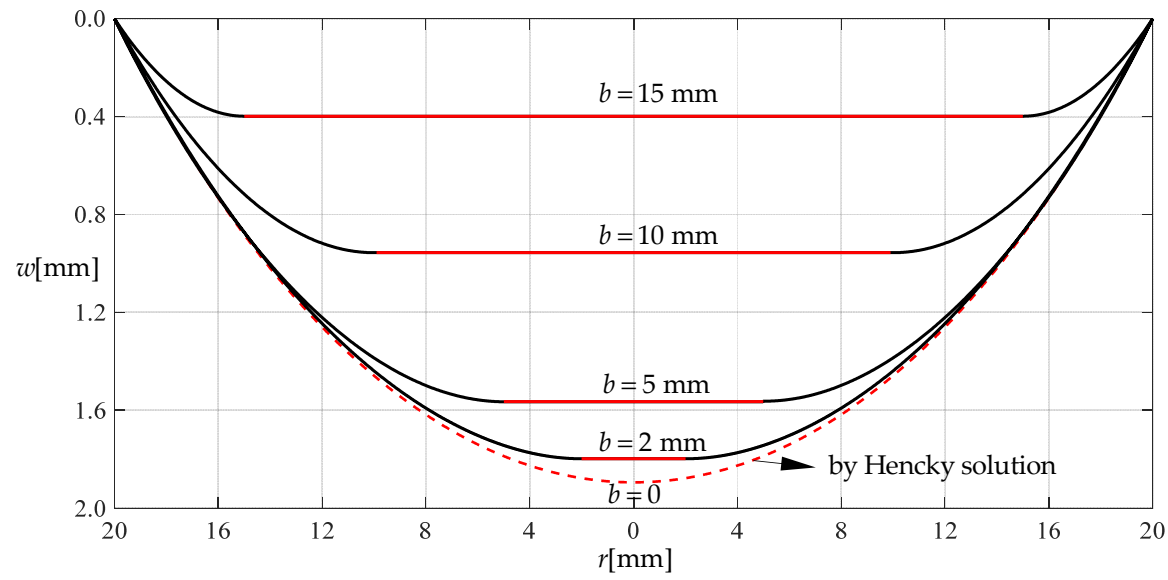

Figure 10. Diagram of the geometry of an annular membrane with a decreasing inner radius $(b=15,10,5$ and $2 \mathrm{~mm})$ gradually approaching the geometry of a circular membrane $(b=0)$ under the same action of the uniformly distributed transverse loads $q=0.0003 \mathrm{MPa}$. 
Lian et al. [28] presented the closed-form solution of the traditional annular membrane structure, the problem of axisymmetric deformation and deflection of an annular membrane with an immovable and indeformable outer edge and a movable but indeformable inner edge connected to a movable but weightless rigid concentric circular thin plate, where both the annular membrane and the movable but weightless rigid concentric circular thin plate are subjected to the uniformly distributed transverse loads $q$. The closed-form solution given in Section 2 is suitable for the new hollow annular membrane structure, the problem of axisymmetric deformation and deflection of an annular membrane with an immovable and indeformable outer edge and a movable but indeformable inner edge attached to a movable but weightless rigid concentric circular ring, where the uniformly distributed transverse loads $q$ is applied only onto the annular membrane. Now, let us compare the closed-form solution presented in this paper with the closed-form solution presented in Lian et al. [28].

The difference between the two closed-form solutions is shown in Figures 11 and 12, where the solid lines represent the hollow annular membrane structure and are calculated by using the closed-form solution presented in this paper, the dash-dot lines represent the traditional annular membrane structure and are calculated by using the closed-form solution presented in Lian et al. [28]. Additionally, the two annular membranes have the same outer radius $a=20 \mathrm{~mm}$ and the same inner radius $b=10 \mathrm{~mm}$.

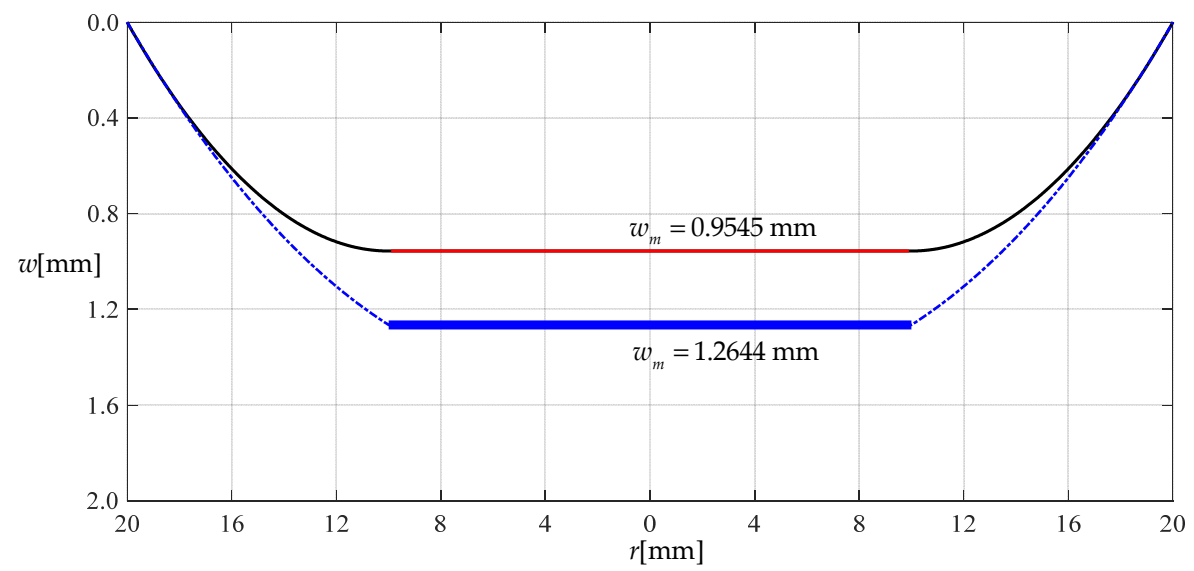

Figure 11. Geometry of two annular membranes with the same inner and outer radii in hollow and traditional annular membrane structures, where the solid line represents the hollow annular membrane structure addressed in this paper and is calculated by using the closed-form solution presented by this paper, the dash-dotted line represents the traditional annular membrane structure addressed in Alekseev [25] and is calculated by using the closed-form solution presented by Lian et al. [28], and the two different annular membrane structures are subjected to the same uniformly distributed transverse loads $q=0.0003 \mathrm{MPa}$, while the two same annular membranes exhibit the different maximum deflections $w_{m}=0.9545 \mathrm{~mm}$ and $1.2644 \mathrm{~mm}$.

Figure 11 shows the difference when the hollow and traditional annular membrane structures are subjected to the same uniformly distributed transverse loads $q=0.0003 \mathrm{MPa}$ while the two same annular membranes exhibit the different maximum deflections $w_{m}=0.9545 \mathrm{~mm}$ and $1.2644 \mathrm{~mm}$. This is because the annular membrane considered in this paper is subjected to the transverse action of $\pi\left(a^{2}-b^{2}\right) q$, while the annular membrane considered in Lian et al. [28] is, due to its movable but indeformable inner edge connected to a movable but weightless rigid concentric circular thin plate, subjected to the transverse action of $\pi a^{2} q$, resulting in the different maximum deflections $w_{m}=0.9545 \mathrm{~mm}$ and $1.2644 \mathrm{~mm}$. According to the so-called conjugate theory of elastic shells that we are working on (the research funded by the National Natural Science Foundation of China, Grant No. 11772072), the two closed-form solutions presented in Lian et al. [28] (the traditional annular membrane structure) and in this paper (the hollow annular membrane structures) can be used to design two kinds of elastic conjugate shells of revolution, respec- 
tively. The closed-form solution of the traditional annular membrane structures can be used to design an elastic conjugate shell of revolution with a movable but indeformable inner edge connected to a movable but weightless rigid concentric circular thin plate. The closed-form solution of the hollow annular membrane structures can be used to design an elastic conjugate shell of revolution with a movable but indeformable inner edge attached to a movable but weightless rigid concentric circular ring. Therefore, the difference in maximum deflections shown in Figure 11 reminds us that the above-mentioned two kinds of elastic conjugate shells of revolution should have different geometry and the maximum height (the rise of shells of revolution, the distance between the base plane (the plane on which the bottom of the shells lies) and apex of the shells), if they have the same inner and outer radii and are subjected to the same uniformly distributed transverse loads $q$.

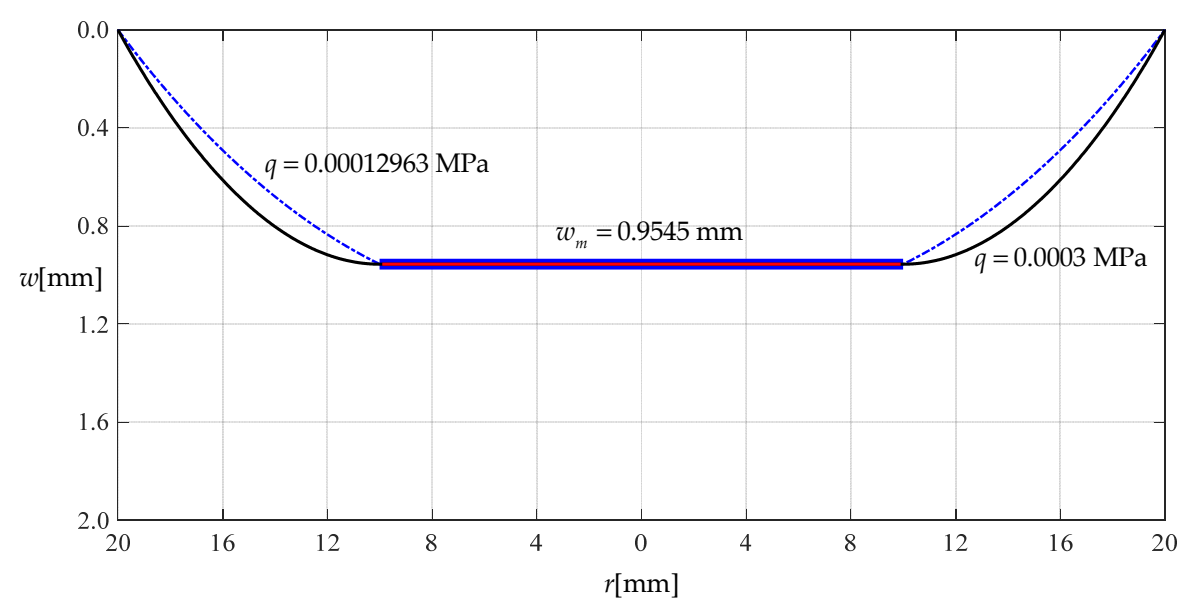

Figure 12. Geometry of two annular membranes with the same inner and outer radii in hollow and traditional annular membrane structures, where the solid line represents the hollow annular membrane structure and is calculated by using the closed-form solution presented by this paper, the dash-dotted line represents the traditional annular membrane structure and is calculated by using the closed-form solution presented by Lian et al. [28], and the two different annular membrane structures are, respectively, subjected to the uniformly distributed transverse loads $q=0.0003 \mathrm{MPa}$ and $0.00012963 \mathrm{MPa}$, while the two same annular membranes exhibit the same maximum deflection $w_{m}=0.9545 \mathrm{~mm}$.

Figure 12 shows the difference when the hollow and traditional annular membrane structures are subjected to the different uniformly distributed transverse loads $q=0.0003 \mathrm{MPa}$ and $0.00012963 \mathrm{MPa}$ while the two same annular membranes exhibit the same maximum deflection $w_{m}=0.9545 \mathrm{~mm}$. However, it may also be seen from Figure 12 that the two annular membranes exhibit the different deflection curve shapes. This to some extent shows that the above-mentioned two kinds of elastic conjugate shells of revolution should have different geometry, if they have the same inner radius, outer radius and maximum height (the rise of shells of revolution).

\section{Concluding Remarks}

In this paper, we deal analytically with the geometrically nonlinear problem of axisymmetric deformation and deflection of a new annular membrane structure subjected to transverse uniform loading. The new structure is an initially flat, linearly elastic, transversely uniformly loaded hollow annular membrane with an immovable and indeformable outer edge and a movable but indeformable inner edge attached to a movable but weightless rigid concentric circular ring. The closed-form solution of the new hollow annular membrane structure is presented. The numerical examples conducted show that the closedform solution presented has satisfactory convergence and is, taking the well-known Hencky solution as the criterion, reliable. 
The traditional annular membrane structure is an annular membrane with an immovable and indeformable outer edge and a movable but indeformable inner edge connected to a movable but weightless rigid concentric circular thin plate. In comparison with the traditional annular membrane structure, the new hollow annular membrane structure lacks the load $\pi b^{2} q$, which is uniformly distributed on the circular thin plate of the traditional annular membrane structure. The load $\pi b^{2} q$ is actually acting on the inner edge of the traditional annular membrane structure and transferred to the annular membrane through its inner edge. Consequently, under the conditions of the same loads $q$ and the same inner and outer radii of annular membrane, the traditional annular membrane structure is actually subjected to a greater external action force $\left(\pi a^{2} q\right)$ than the new hollow annular membrane structure $\left(\pi\left(a^{2}-b^{2}\right) q\right)$, exhibiting a greater maximum deflection. Therefore, the closedform solution suitable for the traditional annular membrane structure does not work with the hollow annular membrane structure. Obviously, the use of the closed-form solution of the traditional annular membrane structure for the hollow annular membrane structure will cause a calculation result larger than the result calculated by using the closed-form solution of the hollow annular membrane structure.

According to the so-called conjugate theory of elastic shells that we are working on (the research funded by the National Natural Science Foundation of China, Grant No. 11772072), the two closed-form solutions of the traditional and hollow annular membrane structures can be used to design two kinds of elastic conjugate shells of revolution, respectively. The closed-form solution of the traditional annular membrane structures can be used to design an elastic conjugate shell of revolution with a movable but indeformable inner edge connected to a movable but weightless rigid concentric circular thin plate. The closedform solution of the hollow annular membrane structures can be used to design an elastic conjugate shell of revolution with a movable but indeformable inner edge attached to a movable but weightless rigid concentric circular ring. Therefore, from the conducted comparison between the two closed-form solutions for the traditional and hollow annular membrane structures, the following conclusions can be drawn. If the above-mentioned two kinds of elastic conjugate shells of revolution have the same inner and outer radii, they should have different geometries and maximum heights (the rise of shells of revolution, the distance between the base plane (the plane on which the bottom of the shells lies) and apex of the shells) if subjected to the same transverse loads $q$, and should have different geometries if they have the same maximum height.

Author Contributions: Conceptualization, J.-Y.S.; methodology, Q.Z., X.L. and J.-Y.S.; validation, X.-T.H. and J.-Y.S.; writing—original draft preparation, Q.Z. and X.L.; writing-review and editing, Q.Z., X.L. and X.-T.H.; visualization, Q.Z. and X.L.; funding acquisition, J.-Y.S. All authors have read and agreed to the published version of the manuscript.

Funding: This research was funded by the National Natural Science Foundation of China (Grant No. 11772072).

Institutional Review Board Statement: Not applicable.

Informed Consent Statement: Not applicable.

Data Availability Statement: Not applicable.

Conflicts of Interest: The authors declare no conflict of interest.

\section{Appendix A}

$$
\begin{aligned}
& c_{2}=-\frac{1}{16 \beta^{4} c_{0}^{2}}\left(Q^{2} \alpha^{4}-2 Q^{2} \alpha^{2} \beta^{2}+Q^{2} \beta^{4}+24 \beta^{3} c_{0}^{2} c_{1}\right), \\
& c_{3}=\frac{1}{48 c_{0}^{3} \beta^{5}}\left(2 Q^{2} \alpha^{4} \beta c_{1}-4 Q^{2} \alpha^{2} \beta^{3} c_{1}+2 Q^{2} \beta^{5} c_{1}+7 Q^{2} \alpha^{4} c_{0}\right. \\
& \left.-10 Q^{2} \alpha^{2} \beta^{2} c_{0}+3 Q^{2} \beta^{4} c_{0}+96 \beta^{3} c_{0}^{3} c_{1}\right),
\end{aligned}
$$




$$
\begin{aligned}
& c_{4}=-\frac{1}{768 \beta^{8} c_{0}{ }^{5}}\left(24 Q^{2} \alpha^{4} \beta^{4} c_{0} c_{1}{ }^{2}-48 Q^{2} \alpha^{2} \beta^{6} c_{0} c_{1}{ }^{2}+24 Q^{2} \beta^{8} c_{0} c_{1}{ }^{2}\right. \\
& +Q^{4} \alpha^{8}-4 Q^{4} \alpha^{6} \beta^{2}+6 Q^{4} \alpha^{4} \beta^{4}-4 Q^{4} \alpha^{2} \beta^{6}+Q^{4} \beta^{8} \\
& +112 Q^{2} \alpha^{4} \beta^{3} c_{0}^{2} c_{1}-160 Q^{2} \alpha^{2} \beta^{5} c_{0}{ }^{2} c_{1}+48 Q^{2} \beta^{7} c_{0} c_{1}+188 Q^{2} \alpha^{4} \beta^{2} c_{0}{ }^{3} \\
& \left.-216 Q^{2} \alpha^{2} \beta^{4} c_{0}{ }^{3}+60 Q^{2} \beta^{6} c_{0}{ }^{3}+1920 \beta^{5} c_{0}{ }^{5} c_{1}\right)
\end{aligned}
$$

$c_{5}=\frac{1}{3840 \beta^{9} c_{0}{ }^{6}}\left(96 Q^{2} \alpha^{4} \beta^{5} c_{0} c_{1}^{3}-192 Q^{2} \alpha^{2} \beta^{7} c_{0} c_{1}^{3}+96 Q^{2} \beta^{9} c_{0} c_{1}^{3}+11 Q^{4} \alpha^{8} \beta c_{1}\right.$

$-44 Q^{4} \alpha^{6} \beta^{3} c_{1}+66 Q^{4} \alpha^{4} \beta^{5} c_{1}-44 Q^{4} \alpha^{2} \beta^{7} c_{1}+11 Q^{4} \beta^{9} c_{1}+576 Q^{2} \alpha^{4} \beta^{4} c_{0}{ }^{2} c_{1}{ }^{2}$

$-864 Q^{2} \alpha^{2} \beta^{6} c_{0}{ }^{2} c_{1}^{2}+288 Q^{2} \beta^{8} c_{0}^{2} c_{1}^{2}+22 Q^{4} \alpha^{8} c_{0}-72 Q^{4} \alpha^{6} \beta^{2} c_{0}+84 Q^{4} \alpha^{4} \beta^{4} c_{0}$

$-40 Q^{4} \alpha^{2} \beta^{6} c_{0}+6 Q^{4} \beta^{8} c_{0}+1272 Q^{2} \alpha^{4} \beta^{3} c_{0}{ }^{3} c_{1}-1392 Q^{2} \alpha^{2} \beta^{5} c_{0}{ }^{3} c_{1}+312 Q^{2} \beta^{7} c_{0}{ }^{3} c_{1}$

$\left.+1368 Q^{2} \alpha^{4} \beta^{2} c_{0}{ }^{4}-1344 Q^{2} \alpha^{2} \beta^{4} c_{0}{ }^{4}+360 Q^{2} \beta^{6} c_{0}{ }^{4}+11520 \beta^{5} c_{0}{ }^{6} c_{1}\right)$

$c_{6}=-\frac{1}{184320 \beta^{12} c_{0}{ }^{8}}\left(3840 Q^{2} \alpha^{4} \beta^{8} c_{0}{ }^{2} c_{1}{ }^{4}-7680 Q^{2} \alpha^{2} \beta^{10} c_{0}{ }^{2} c_{1}{ }^{4}+3840 Q^{2} \beta^{12} c_{0}{ }^{2} c_{1}{ }^{4}\right.$

$+816 Q^{4} \alpha^{8} \beta^{4} c_{0} c_{1}^{2}-3264 Q^{4} \alpha^{6} \beta^{6} c_{0} c_{1}{ }^{2}+4896 Q^{4} \alpha^{4} \beta^{8} c_{0} c_{1}{ }^{2}-3264 Q^{4} \alpha^{2} \beta^{10} c_{0} c_{1}{ }^{2}$

$+816 Q^{4} \beta^{12} c_{0} c_{1}^{2}+28416 Q^{2} \alpha^{4} \beta^{7} c_{0}{ }^{3} c_{1}{ }^{3}-44544 Q^{2} \alpha^{2} \beta^{9} c_{0}{ }^{3} c_{1}{ }^{3}+16128 Q^{2} \beta^{11} c_{0}{ }^{3} c_{1}{ }^{3}$

$+11 Q^{6} \alpha^{12}-66 Q^{6} \alpha^{10} \beta^{2}+165 Q^{6} \alpha^{8} \beta^{4}-220 Q^{6} \alpha^{6} \beta^{6}+165 Q^{6} \alpha^{4} \beta^{8}-66 Q^{6} \alpha^{2} \beta^{10}$

$+11 Q^{6} \beta^{12}+3000 Q^{4} \alpha^{8} \beta^{3} c_{0}^{2} c_{1}-10080 Q^{4} \alpha^{6} \beta^{5} c_{0}^{2} c_{1}+12240 Q^{4} \alpha^{4} \beta^{7} c_{0}{ }^{2} c_{1}$

$-6240 Q^{4} \alpha^{2} \beta^{9} c_{0}{ }^{2} c_{1}+1080 Q^{4} \beta^{11} c_{0}{ }^{2} c_{1}+81216 Q^{2} \alpha^{4} \beta^{6} c_{0}{ }^{4} c_{1}{ }^{2}-95616 Q^{2} \alpha^{2} \beta^{8} c_{0}{ }^{4} c_{1}{ }^{2}$

$+23616 Q^{2} \beta^{10} c_{0}{ }^{4} c_{1}{ }^{2}+2856 Q^{4} \alpha^{8} \beta^{2} c_{0}{ }^{3}-7968 Q^{4} \alpha^{6} \beta^{4} c_{0}{ }^{3}+7728 Q^{4} \alpha^{4} \beta^{6} c_{0}{ }^{3}$

$-2976 Q^{4} \alpha^{2} \beta^{8} c_{0}{ }^{3}+360 Q^{4} \beta^{10} c_{0}{ }^{3}+113472 Q^{2} \alpha^{4} \beta^{5} c_{0}{ }^{5} c_{1}-99456 Q^{2} \alpha^{2} \beta^{7} c_{0}{ }^{5} c_{1}$

$+18240 Q^{2} \beta^{9} c_{0}{ }^{5} c_{1}+88128 Q^{2} \alpha^{4} \beta^{4} c_{0}{ }^{6}-76800 Q^{2} \alpha^{2} \beta^{6} c_{0}{ }^{6}+20160 Q^{2} \beta^{8} c_{0}{ }^{6}$

$\left.+645120 \beta^{7} c_{0}^{8} c_{1}\right)$

$c_{7}=-\frac{1}{42 \beta^{4} c_{0}^{2}}\left(60 \beta^{4} c_{0} c_{1} c_{6}+44 \beta^{4} c_{0} c_{2} c_{5}+36 \beta^{4} c_{0} c_{3} c_{4}+20 \beta^{4} c_{1}^{2} c_{5}+28 \beta^{4} c_{1} c_{2} c_{4}\right.$

$+12 \beta^{4} c_{1} c_{3}^{2}+10 \beta^{4} c_{2}{ }^{2} c_{3}+138 \beta^{3} c_{0}{ }^{2} c_{6}+196 \beta^{3} c_{0} c_{1} c_{5}+148 \beta^{3} c_{0} c_{2} c_{4}+66 \beta^{3} c_{0} c_{3}^{2}$

$+66 \beta^{3} c_{1}^{2} c_{4}+100 \beta^{3} c_{1} c_{2} c_{3}+14 \beta^{3} c_{2}{ }^{3}+165 \beta^{2} c_{0}{ }^{2} c_{5}+234 \beta^{2} c_{0} c_{1} c_{4}+186 \beta^{2} c_{0} c_{2} c_{3}{ }^{2} c^{3}$

$+81 \beta^{2} c_{1}^{2} c_{3}+69 \beta^{2} c_{1} c_{2}^{2}+84 \beta c_{0}^{2} c_{4}+120 \beta c_{0} c_{1} c_{3}+52 \beta c_{0} c_{2}^{2}+44 \beta c_{1}^{2} c_{2}$

$\left.+15 c_{0}^{2} c_{3}+22 c_{0} c_{1} c_{2}+3 c_{1}^{3}\right)$

$c_{8}=-\frac{1}{56 \beta^{4} c_{0}{ }^{2}}\left(84 \beta^{4} c_{0} c_{1} c_{7}+64 \beta^{4} c_{0} c_{2} c_{6}+52 \beta^{4} c_{0} c_{3} c_{5}+24 \beta^{4} c_{0} c_{4}{ }^{2}+30 \beta^{4} c_{1}{ }^{2} c_{6}\right.$

$+44 \beta^{4} c_{1} c_{2} c_{5}+36 \beta^{4} c_{1} c_{3} c_{4}+16 \beta^{4} c_{2}^{2} c_{4}+14 \beta^{4} c_{2} c_{3}^{2}+189 \beta^{3} c_{0}{ }^{2} c_{7}+282 \beta^{3} c_{0} c_{1} c_{6}$

$+218 \beta^{3} c_{0} c_{2} c_{5}+186 \beta^{3} c_{0} c_{3} c_{4}+101 \beta^{3} c_{1}^{2} c_{5}+154 \beta^{3} c_{1} c_{2} c_{4}+69 \beta^{3} c_{1} c_{3}^{2}+61 \beta^{3} c_{2}^{2} c_{3}$,

$+234 \beta^{2} c_{0}^{2} c_{6}+348 \beta^{2} c_{0} c_{1} c_{5}+276 \beta^{2} c_{0} c_{2} c_{4}+126 \beta^{2} c_{0} c_{3}^{2}+126 \beta^{2} c_{1}^{2} c_{4}$

$+204 \beta^{2} c_{1} c_{2} c_{3}+30 \beta^{2} c_{2}^{3}+125 \beta c_{0}^{2} c_{5}+186 \beta c_{0} c_{1} c_{4}+154 \beta c_{0} c_{2} c_{3}+69 \beta c_{1}^{2} c_{3}$

$\left.+61 \beta c_{1} c_{2}^{2}+24 c_{0}^{2} c_{4}+36 c_{0} c_{1} c_{3}+16 c_{0} c_{2}^{2}+14 c_{1}^{2} c_{2}\right)$

$c_{9}=-\frac{1}{72 \beta^{4} c_{0}^{2}}\left(112 \beta^{4} c_{0} c_{1} c_{8}+88 \beta^{4} c_{0} c_{2} c_{7}+72 \beta^{4} c_{0} c_{3} c_{6}+64 \beta^{4} c_{0} c_{4} c_{5}+42 \beta^{4} c_{1}^{2} c_{7}\right.$

$+64 \beta^{4} c_{1} c_{2} c_{6}+52 \beta^{4} c_{1} c_{3} c_{5}+24 \beta^{4} c_{1} c_{4}^{2}+24 \beta^{4} c_{2}{ }^{2} c_{5}+40 \beta^{4} c_{2} c_{3} c_{4}+6 \beta^{4} c_{3}^{3}$

$+248 \beta^{3} c_{0}^{2} c_{8}+384 \beta^{3} c_{0} c_{1} c_{7}+304 \beta^{3} c_{0} c_{2} c_{6}+256 \beta^{3} c_{0} c_{3} c_{5}+120 \beta^{3} c_{0} c_{4}^{2}$

$+144 \beta^{3} c_{1}^{2} c_{6}+224 \beta^{3} c_{1} c_{2} c_{5}+192 \beta^{3} c_{1} c_{3} c_{4}+88 \beta^{3} c_{2}{ }^{2} c_{4}+80 \beta^{3} c_{2} c_{3}^{2}+315 \beta^{2} c_{0}^{2} c_{7}$ ，

$+486 \beta^{2} c_{0} c_{1} c_{6}+390 \beta^{2} c_{0} c_{2} c_{5}+342 \beta^{2} c_{0} c_{3} c_{4}+183 \beta^{2} c_{1}^{2} c_{5}+294 \beta^{2} c_{1} c_{2} c_{4}$

$+135 \beta^{2} c_{1} c_{3}^{2}+123 \beta^{2} c_{2}^{2} c_{3}+174 \beta c_{0}^{2} c_{6}+268 \beta c_{0} c_{1} c_{5}+220 \beta c_{0} c_{2} c_{4}+102 \beta c_{0} c_{3}{ }^{2}$

$+102 \beta c_{1}^{2} c_{4}+172 \beta c_{1} c_{2} c_{3}+26 \beta c_{2}^{3}+35 c_{0}^{2} c_{5}+54 c_{0} c_{1} c_{4}+46 c_{0} c_{2} c_{3}+21 c_{1}^{2} c_{3}$

$\left.+19 c_{1} c_{2}^{2}\right)$ 


$$
\begin{aligned}
& c_{10}=-\frac{1}{90 \beta^{4} c_{0}^{2}}\left(144 \beta^{4} c_{0} c_{1} c_{9}+116 \beta^{4} c_{0} c_{2} c_{8}+96 \beta^{4} c_{0} c_{3} c_{7}+84 \beta^{4} c_{0} c_{4} c_{6}\right. \\
& +40 \beta^{4} c_{0} c_{5}^{2}+56 \beta^{4} c_{1}^{2} c_{8}+88 \beta^{4} c_{1} c_{2} c_{7}+72 \beta^{4} c_{1} c_{3} c_{6}+64 \beta^{4} c_{1} c_{4} c_{5}+34 \beta^{4} c_{2}{ }^{2} c_{6} \\
& +56 \beta^{4} c_{2} c_{3} c_{5}+26 \beta^{4} c_{2} c_{4}^{2}+24 \beta^{4} c_{3}^{2} c_{4}+315 \beta^{3} c_{0}^{2} c_{9}+502 \beta^{3} c_{0} c_{1} c_{8}+406 \beta^{3} c_{0} c_{2} c_{7} \\
& +342 \beta^{3} c_{0} c_{3} c_{6}+310 \beta^{3} c_{0} c_{4} c_{5}+195 \beta^{3} c_{1}^{2} c_{7}+310 \beta^{3} c_{1} c_{2} c_{6}+262 \beta^{3} c_{1} c_{3} c_{5} \\
& +123 \beta^{3} c_{1} c_{4}^{2}+123 \beta^{3} c_{2}^{2} c_{5}+214 \beta^{3} c_{2} c_{3} c_{4}+33 \beta^{3} c_{3}{ }^{3}+408 \beta^{2} c_{0}{ }^{2} c_{8}+648 \beta^{2} c_{0} c_{1} c_{7} \\
& +528 \beta^{2} c_{0} c_{2} c_{6}+456 \beta^{2} c_{0} c_{3} c_{5}+216 \beta^{2} c_{0} c_{4}^{2}+252 \beta^{2} c_{1}^{2} c_{6}+408 \beta^{2} c_{1} c_{2} c_{5} \\
& +360 \beta^{2} c_{1} c_{3} c_{4}+168 \beta^{2} c_{2}^{2} c_{4}+156 \beta^{2} c_{2} c_{3}^{2}+231 \beta c_{0}^{2} c_{7}+366 \beta c_{0} c_{1} c_{6}+302 \beta c_{0} c_{2} c_{5} \\
& +270 \beta c_{0} c_{3} c_{4}+143 \beta c_{1}^{2} c_{5}+238 \beta c_{1} c_{2} c_{4}+111 \beta c_{1} c_{3}^{2}+103 \beta c_{2}^{2} c_{3}+48 c_{0}^{2} c_{6} \\
& \left.+76 c_{0} c_{1} c_{5}+64 c_{0} c_{2} c_{4}+30 c_{0} c_{3}^{2}+30 c_{1}^{2} c_{4}+52 c_{1} c_{2} c_{3}+8 c_{2}^{3}\right) \\
& c_{11}=-\frac{1}{110 \beta^{4} c_{0}^{2}}\left(180 \beta^{4} c_{0} c_{1} c_{10}+148 \beta^{4} c_{0} c_{2} c_{9}+124 \beta^{4} c_{0} c_{3} c_{8}+108 \beta^{4} c_{0} c_{4} c_{7}\right. \\
& +100 \beta^{4} c_{0} c_{5} c_{6}+72 \beta^{4} c_{1}^{2} c_{9}+116 \beta^{4} c_{1} c_{2} c_{8}+96 \beta^{4} c_{1} c_{3} c_{7}+84 \beta^{4} c_{1} c_{4} c_{6}+40 \beta^{4} c_{1} c_{5}{ }^{2} \\
& +46 \beta^{4} c_{2}{ }^{2} c_{7}+76 \beta^{4} c_{2} c_{3} c_{6}+68 \beta^{4} c_{2} c_{4} c_{5}+32 \beta^{4} c_{3}{ }^{2} c_{5}+30 \beta^{4} c_{3} c_{4}{ }^{2}+390 \beta^{3} c_{0}{ }^{2} c_{10} \\
& +636 \beta^{3} c_{0} c_{1} c_{9}+524 \beta^{3} c_{0} c_{2} c_{8}+444 \beta^{3} c_{0} c_{3} c_{7}+396 \beta^{3} c_{0} c_{4} c_{6}+190 \beta^{3} c_{0} c_{5}{ }^{2} \\
& +254 \beta^{3} c_{1}^{2} c_{8}+412 \beta^{3} c_{1} c_{2} c_{7}+348 \beta^{3} c_{1} c_{3} c_{6}+316 \beta^{3} c_{1} c_{4} c_{5}+166 \beta^{3} c_{2}{ }^{2} c_{6} \\
& +284 \beta^{3} c_{2} c_{3} c_{5}+134 \beta^{3} c_{2} c_{4}^{2}+126 \beta^{3} c_{3}^{2} c_{4}+513 \beta^{2} c_{0}{ }^{2} c_{9}+834 \beta^{2} c_{0} c_{1} c_{8} \\
& +690 \beta^{2} c_{0} c_{2} c_{7}+594 \beta^{2} c_{0} c_{3} c_{6}+546 \beta^{2} c_{0} c_{4} c_{5}+333 \beta^{2} c_{1}^{2} c_{7}+546 \beta^{2} c_{1} c_{2} c_{6} \\
& +474 \beta^{2} c_{1} c_{3} c_{5}+225 \beta^{2} c_{1} c_{4}^{2}+225 \beta^{2} c_{2}^{2} c_{5}+402 \beta^{2} c_{2} c_{3} c_{4}+63 \beta^{2} c_{3}^{3}+296 \beta c_{0}^{2} c_{8} \\
& +480 \beta c_{0} c_{1} c_{7}+400 \beta c_{0} c_{2} c_{6}+352 \beta c_{0} c_{3} c_{5}+168 \beta c_{0} c_{4}^{2}+192 \beta c_{1}^{2} c_{6}+320 \beta c_{1} c_{2} c_{5} \\
& +288 \beta c_{1} c_{3} c_{4}+136 \beta c_{2}^{2} c_{4}+128 \beta c_{2} c_{3}^{2}+63 c_{0}^{2} c_{7}+102 c_{0} c_{1} c_{6}+86 c_{0} c_{2} c_{5} \\
& \left.+78 c_{0} c_{3} c_{4}+41 c_{1}^{2} c_{5}+70 c_{1} c_{2} c_{4}+33 c_{1} c_{3}^{2}+31 c_{2}^{2} c_{3}\right) \\
& c_{12}=-\frac{1}{132 \beta^{4} c_{0}{ }^{2}}\left(220 \beta^{4} c_{0} c_{1} c_{11}+184 \beta^{4} c_{0} c_{2} c_{10}+156 \beta^{4} c_{0} c_{3} c_{9}+136 \beta^{4} c_{0} c_{4} c_{8}\right. \\
& +124 \beta^{4} c_{0} c_{5} c_{7}+60 \beta^{4} c_{0} c_{6}{ }^{2}+90 \beta^{4} c_{1}{ }^{2} c_{10}+148 \beta^{4} c_{1} c_{2} c_{9}+124 \beta^{4} c_{1} c_{3} c_{8} \\
& +108 \beta^{4} c_{1} c_{4} c_{7}+100 \beta^{4} c_{1} c_{5} c_{6}+60 \beta^{4} c_{2}{ }^{2} c_{8}+100 \beta^{4} c_{2} c_{3} c_{7}+88 \beta^{4} c_{2} c_{4} c_{6} \\
& +42 \beta^{4} c_{2} c_{5}{ }^{2}+42 \beta^{4} c_{3}{ }^{2} c_{6}+76 \beta^{4} c_{3} c_{4} c_{5}+12 \beta^{4} c_{4}{ }^{3}+473 \beta^{3} c_{0}{ }^{2} c_{11}+786 \beta^{3} c_{0} c_{1} c_{10} \\
& +658 \beta^{3} c_{0} c_{2} c_{9}+562 \beta^{3} c_{0} c_{3} c_{8}+498 \beta^{3} c_{0} c_{4} c_{7}+466 \beta^{3} c_{0} c_{5} c_{6}+321 \beta^{3} c_{1}^{2} c_{9} \\
& +530 \beta^{3} c_{1} c_{2} c_{8}+450 \beta^{3} c_{1} c_{3} c_{7}+402 \beta^{3} c_{1} c_{4} c_{6}+193 \beta^{3} c_{1} c_{5}^{2}+217 \beta^{3} c_{2}{ }^{2} c_{7} \\
& +370 \beta^{3} c_{2} c_{3} c_{6}+338 \beta^{3} c_{2} c_{4} c_{5}+161 \beta^{3} c_{3}^{2} c_{5}+153 \beta^{3} c_{3} c_{4}^{2}+630 \beta^{2} c_{0}^{2} c_{10} \\
& +1044 \beta^{2} c_{0} c_{1} c_{9}+876 \beta^{2} c_{0} c_{2} c_{8}+756 \beta^{2} c_{0} c_{3} c_{7}+684 \beta^{2} c_{0} c_{4} c_{6}+330 \beta^{2} c_{0} c_{5}{ }^{2} \\
& +426 \beta^{2} c_{1}^{2} c_{8}+708 \beta^{2} c_{1} c_{2} c_{7}+612 \beta^{2} c_{1} c_{3} c_{6}+564 \beta^{2} c_{1} c_{4} c_{5}+294 \beta^{2} c_{2}^{2} c_{6} \\
& +516 \beta^{2} c_{2} c_{3} c_{5}+246 \beta^{2} c_{2} c_{4}^{2}+234 \beta^{2} c_{3}^{2} c_{4}+369 \beta c_{0}^{2} c_{9}+610 \beta c_{0} c_{1} c_{8} \\
& +514 \beta c_{0} c_{2} c_{7}+450 \beta c_{0} c_{3} c_{6}+418 \beta c_{0} c_{4} c_{5}+249 \beta c_{1}^{2} c_{7}+418 \beta c_{1} c_{2} c_{6}+370 \beta c_{1} c_{3} c_{5} \\
& +177 \beta c_{1} c_{4}^{2}+177 \beta c_{2}^{2} c_{5}+322 \beta c_{2} c_{3} c_{4}+51 \beta c_{3}{ }^{3}+80 c_{0}^{2} c_{8}+132 c_{0} c_{1} c_{7}+112 c_{0} c_{2} c_{6} \\
& \left.+100 c_{0} c_{3} c_{5}+48 c_{0} c_{4}^{2}+54 c_{1}^{2} c_{6}+92 c_{1} c_{2} c_{5}+84 c_{1} c_{3} c_{4}+40 c_{2}^{2} c_{4}+38 c_{2} c_{3}^{2}\right) \\
& c_{13}=-\frac{1}{156 \beta^{4} c_{0}{ }^{2}}\left(264 \beta^{4} c_{0} c_{1} c_{12}+224 \beta^{4} c_{0} c_{2} c_{11}+192 \beta^{4} c_{0} c_{3} c_{10}+168 \beta^{4} c_{0} c_{4} c_{9}\right. \\
& +152 \beta^{4} c_{0} c_{5} c_{8}+144 \beta^{4} c_{0} c_{6} c_{7}+110 \beta^{4} c_{1}^{2} c_{11}+184 \beta^{4} c_{1} c_{2} c_{10}+156 \beta^{4} c_{1} c_{3} c_{9} \\
& +136 \beta^{4} c_{1} c_{4} c_{8}+124 \beta^{4} c_{1} c_{5} c_{7}+60 \beta^{4} c_{1} c_{6}{ }^{2}+76 \beta^{4} c_{2}{ }^{2} c_{9}+128 \beta^{4} c_{2} c_{3} c_{8} \\
& +112 \beta^{4} c_{2} c_{4} c_{7}+104 \beta^{4} c_{2} c_{5} c_{6}+54 \beta^{4} c_{3}{ }^{2} c_{7}+96 \beta^{4} c_{3} c_{4} c_{6}+46 \beta^{4} c_{3} c_{5}{ }^{2} \\
& +44 \beta^{4} c_{4}^{2} c_{5}+564 \beta^{3} c_{0}^{2} c_{12}+952 \beta^{3} c_{0} c_{1} c_{11}+808 \beta^{3} c_{0} c_{2} c_{10}+696 \beta^{3} c_{0} c_{3} c_{9} \\
& +616 \beta^{3} c_{0} c_{4} c_{8}+568 \beta^{3} c_{0} c_{5} c_{7}+276 \beta^{3} c_{0} c_{6}^{2}+396 \beta^{3} c_{1}^{2} c_{10}+664 \beta^{3} c_{1} c_{2} c_{9} \\
& +568 \beta^{3} c_{1} c_{3} c_{8}+504 \beta^{3} c_{1} c_{4} c_{7}+472 \beta^{3} c_{1} c_{5} c_{6}+276 \beta^{3} c_{2}{ }^{2} c_{8}+472 \beta^{3} c_{2} c_{3} c_{7} \\
& +424 \beta^{3} c_{2} c_{4} c_{6}+204 \beta^{3} c_{2} c_{5}^{2}+204 \beta^{3} c_{3}^{2} c_{6}+376 \beta^{3} c_{3} c_{4} c_{5}+60 \beta^{3} c_{4}{ }^{3} \\
& +759 \beta^{2} c_{0}^{2} c_{11}+1278 \beta^{2} c_{0} c_{1} c_{10}+1086 \beta^{2} c_{0} c_{2} c_{9}+942 \beta^{2} c_{0} c_{3} c_{8} \\
& +846 \beta^{2} c_{0} c_{4} c_{7}+798 \beta^{2} c_{0} c_{5} c_{6}+531 \beta^{2} c_{1}^{2} c_{9}+894 \beta^{2} c_{1} c_{2} c_{8}+774 \beta^{2} c_{1} c_{3} c_{7} \\
& +702 \beta^{2} c_{1} c_{4} c_{6}+339 \beta^{2} c_{1} c_{5}^{2}+375 \beta^{2} c_{2}^{2} c_{7}+654 \beta^{2} c_{2} c_{3} c_{6}+606 \beta^{2} c_{2} c_{4} c_{5} \\
& +291 \beta^{2} c_{3}^{2} c_{5}+279 \beta^{2} c_{3} c_{4}^{2}+450 \beta c_{0}^{2} c_{10}+756 \beta c_{0} c_{1} c_{9}+644 \beta c_{0} c_{2} c_{8}
\end{aligned}
$$




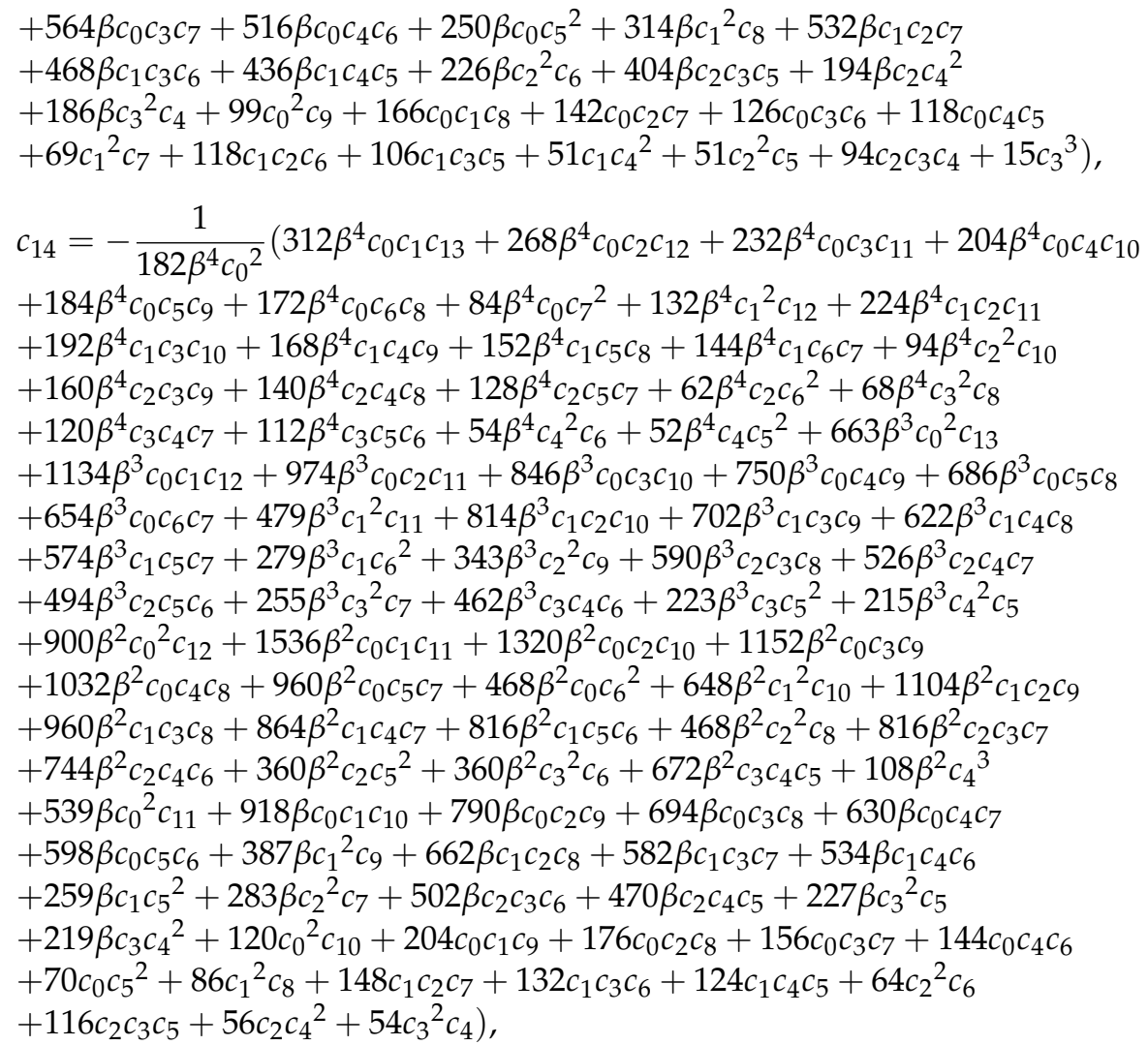

$c_{15}=-\frac{1}{210 \beta^{4} c_{0}{ }^{2}}\left(364 \beta^{4} c_{0} c_{1} c_{14}+316 \beta^{4} c_{0} c_{2} c_{13}+276 \beta^{4} c_{0} c_{3} c_{12}+244 \beta^{4} c_{0} c_{4} c_{11}\right.$ $+220 \beta^{4} c_{0} c_{5} c_{10}+204 \beta^{4} c_{0} c_{6} c_{9}+196 \beta^{4} c_{0} c_{7} c_{8}+156 \beta^{4} c_{1}{ }^{2} c_{13}+268 \beta^{4} c_{1} c_{2} c_{12}$ $+232 \beta^{4} c_{1} c_{3} c_{11}+204 \beta^{4} c_{1} c_{4} c_{10}+184 \beta^{4} c_{1} c_{5} c_{9}+172 \beta^{4} c_{1} c_{6} c_{8}+84 \beta^{4} c_{1} c_{7}{ }^{2}$ $+114 \beta^{4} c_{2}^{2} c_{11}+196 \beta^{4} c_{2} c_{3} c_{10}+172 \beta^{4} c_{2} c_{4} c_{9}+156 \beta^{4} c_{2} c_{5} c_{8}+148 \beta^{4} c_{2} c_{6} c_{7}$ $+84 \beta^{4} c_{3}^{2} c_{9}+148 \beta^{4} c_{3} c_{4} c_{8}+136 \beta^{4} c_{3} c_{5} c_{7}+66 \beta^{4} c_{3} c_{6}{ }^{2}+66 \beta^{4} c_{4}{ }^{2} c_{7}$ $+124 \beta^{4} c_{4} c_{5} c_{6}+20 \beta^{4} c_{5}{ }^{3}+770 \beta^{3} c_{0}{ }^{2} c_{14}+1332 \beta^{3} c_{0} c_{1} c_{13}+1156 \beta^{3} c_{0} c_{2} c_{12}$ $+1012 \beta^{3} c_{0} c_{3} c_{11}+900 \beta^{3} c_{0} c_{4} c_{10}+820 \beta^{3} c_{0} c_{5} c_{9}+772 \beta^{3} c_{0} c_{6} c_{8}+378 \beta^{3} c_{0} c_{7}{ }^{2}$ $+570 \beta^{3} c_{1}^{2} c_{12}+980 \beta^{3} c_{1} c_{2} c_{11}+852 \beta^{3} c_{1} c_{3} c_{10}+756 \beta^{3} c_{1} c_{4} c_{9}+692 \beta^{3} c_{1} c_{5} c_{8}$ $+660 \beta^{3} c_{1} c_{6} c_{7}+418 \beta^{3} c_{2}^{2} c_{10}+724 \beta^{3} c_{2} c_{3} c_{9}+644 \beta^{3} c_{2} c_{4} c_{8}+596 \beta^{3} c_{2} c_{5} c_{7}$ $+290 \beta^{3} c_{2} c_{6}{ }^{2}+314 \beta^{3} c_{3}{ }^{2} c_{8}+564 \beta^{3} c_{3} c_{4} c_{7}+532 \beta^{3} c_{3} c_{5} c_{6}+258 \beta^{3} c_{4}{ }^{2} c_{6}$ $+250 \beta^{3} c_{4} c_{5}^{2}+1053 \beta^{2} c_{0}^{2} c_{13}+1818 \beta^{2} c_{0} c_{1} c_{12}+1578 \beta^{2} c_{0} c_{2} c_{11}$ $+1386 \beta^{2} c_{0} c_{3} c_{10}+1242 \beta^{2} c_{0} c_{4} c_{9}+1146 \beta^{2} c_{0} c_{5} c_{8}+1098 \beta^{2} c_{0} c_{6} c_{7}$ $+777 \beta^{2} c_{1}^{2} c_{11}+1338 \beta^{2} c_{1} c_{2} c_{10}+1170 \beta^{2} c_{1} c_{3} c_{9}+1050 \beta^{2} c_{1} c_{4} c_{8}$ $+978 \beta^{2} c_{1} c_{5} c_{7}+477 \beta^{2} c_{1} c_{6}^{2}+573 \beta^{2} c_{2}^{2} c_{9}+1002 \beta^{2} c_{2} c_{3} c_{8}+906 \beta^{2} c_{2} c_{4} c_{7}$ $+858 \beta^{2} c_{2} c_{5} c_{6}+441 \beta^{2} c_{3}{ }^{2} c_{7}+810 \beta^{2} c_{3} c_{4} c_{6}+393 \beta^{2} c_{3} c_{5}^{2}+381 \beta^{2} c_{4}^{2} c_{5}$ $+636 \beta c_{0}^{2} c_{12}+1096 \beta c_{0} c_{1} c_{11}+952 \beta c_{0} c_{2} c_{10}+840 \beta c_{0} c_{3} c_{9}+760 \beta c_{0} c_{4} c_{8}$ $+712 \beta c_{0} c_{5} c_{7}+348 \beta c_{0} c_{6}^{2}+468 \beta c_{1}^{2} c_{10}+808 \beta c_{1} c_{2} c_{9}+712 \beta c_{1} c_{3} c_{8}$ $+648 \beta c_{1} c_{4} c_{7}+616 \beta c_{1} c_{5} c_{6}+348 \beta c_{2}{ }^{2} c_{8}+616 \beta c_{2} c_{3} c_{7}+568 \beta c_{2} c_{4} c_{6}$ $+276 \beta c_{2} c_{5}^{2}+276 \beta c_{3}^{2} c_{6}+520 \beta c_{3} c_{4} c_{5}+84 \beta c_{4}^{3}+143 c_{0}^{2} c_{11}+246 c_{0} c_{1} c_{10}$ $+214 c_{0} c_{2} c_{9}+190 c_{0} c_{3} c_{8}+174 c_{0} c_{4} c_{7}+166 c_{0} c_{5} c_{6}+105 c_{1}^{2} c_{9}+182 c_{1} c_{2} c_{8}$ $+162 c_{1} c_{3} c_{7}+150 c_{1} c_{4} c_{6}+73 c_{1} c_{5}^{2}+79 c_{2}{ }^{2} c_{7}+142 c_{2} c_{3} c_{6}+134 c_{2} c_{4} c_{5}$ $\left.+65 c_{3}^{2} c_{5}+63 c_{3} c_{4}^{2}\right)$ 


\section{Appendix B}

$$
\begin{aligned}
& d_{1}=\frac{Q}{2 \beta c_{0}}\left(\alpha^{2}-\beta^{2}\right) \\
& d_{2}=-\frac{Q}{4 \beta^{2} c_{0}^{2}}\left(\alpha^{2} \beta c_{1}-\beta^{3} c_{1}+\alpha^{2} c_{0}+\beta^{2} c_{0}\right) \text {, } \\
& d_{3}=\frac{Q}{96 \beta^{5} c_{0}{ }^{4}}\left(16 \alpha^{2} \beta^{4} c_{0} c_{1}^{2}-16 \beta^{6} c_{0} c_{1}^{2}+Q^{2} \alpha^{6}-3 Q^{2} \alpha^{4} \beta^{2}+3 Q^{2} \alpha^{2} \beta^{4}\right. \\
& \left.-Q^{2} \beta^{6}+40 \alpha^{2} \beta^{3} c_{0}^{2} c_{1}-8 \beta^{5} c_{0}^{2} c_{1}+16 \alpha^{2} \beta^{2} c_{0}^{3}\right) \\
& d_{4}=-\frac{Q}{192 \beta^{6} c_{0}{ }^{5}}\left(24 \alpha^{2} \beta^{5} c_{0} c_{1}^{3}-24 \beta^{7} c_{0} c_{1}^{3}+4 Q^{2} \alpha^{6} \beta c_{1}-12 Q^{2} \alpha^{4} \beta^{3} c_{1}\right. \\
& +12 Q^{2} \alpha^{2} \beta^{5} c_{1}-4 Q^{2} \beta^{7} c_{1}+96 \alpha^{2} \beta^{4} c_{0}{ }^{2} c_{1}^{2}-48 \beta^{6} c_{0}^{2} c_{1}^{2}+5 Q^{2} \alpha^{6} c_{0} \quad, \\
& \left.-10 Q^{2} \alpha^{4} \beta^{2} c_{0}+5 Q^{2} \alpha^{2} \beta^{4} c_{0}+108 \alpha^{2} \beta^{3} c_{0}{ }^{3} c_{1}-12 \beta^{5} c_{0}{ }^{3} c_{1}+24 \alpha^{2} \beta^{2} c_{0}{ }^{4}\right) \\
& d_{5}=\frac{Q}{1920 \beta^{9} c_{0}{ }^{7}}\left(192 \alpha^{2} \beta^{8} c_{0}{ }^{2} c_{1}{ }^{4}-192 \beta^{10} c_{0}{ }^{2} c_{1}{ }^{4}+58 Q^{2} \alpha^{6} \beta^{4} c_{0} c_{1}{ }^{2}-174 Q^{2} \alpha^{4} \beta^{6} c_{0} c_{1}{ }^{2}\right. \\
& +174 Q^{2} \alpha^{2} \beta^{8} c_{0} c_{1}{ }^{2}-58 Q^{2} \beta^{10} c_{0} c_{1}{ }^{2}+1056 \alpha^{2} \beta^{7} c_{0}{ }^{3} c_{1}{ }^{3}-672 \beta^{9} c_{0}{ }^{3} c_{1}{ }^{3}+Q^{4} \alpha^{10} \\
& -5 Q^{4} \alpha^{8} \beta^{2}+10 Q^{4} \alpha^{6} \beta^{4}-10 Q^{4} \alpha^{4} \beta^{6}+5 Q^{4} \alpha^{2} \beta^{8}-Q^{4} \beta^{10}+152 Q^{2} \alpha^{6} \beta^{3} c_{0}{ }^{2} c_{1} \\
& -344 Q^{2} \alpha^{4} \beta^{5} c_{0}{ }^{2} c_{1}+232 Q^{2} \alpha^{2} \beta^{7} c_{0}^{2} c_{1}-40 Q^{2} \beta^{9} c_{0}{ }^{2} c_{1}+1968 \alpha^{2} \beta^{6} c_{0}{ }^{4} c_{1}{ }^{2} \\
& -624 \beta^{8} c_{0}{ }^{4} c_{1}{ }^{2}+87 Q^{2} \alpha^{6} \beta^{2} c_{0}{ }^{3}-137 Q^{2} \alpha^{4} \beta^{4} c_{0}{ }^{3}+53 Q^{2} \alpha^{2} \beta^{6} c_{0}{ }^{3}-3 Q^{2} \beta^{8} c_{0}{ }^{3} \\
& \left.+1344 \alpha^{2} \beta^{5} c_{0}{ }^{5} c_{1}-96 \beta^{7} c_{0}{ }^{5} c_{1}+192 \alpha^{2} \beta^{4} c_{0}{ }^{6}\right) \\
& d_{6}=-\frac{Q}{23040 \beta^{10} c_{0}{ }^{8}}\left(1920 \alpha^{2} \beta^{9} c_{0}{ }^{2} c_{1}{ }^{5}-1920 \beta^{11} c_{0}{ }^{2} c_{1}{ }^{5}+888 Q^{2} \alpha^{6} \beta^{5} c_{0} c_{1}^{3}\right. \\
& -2664 Q^{2} \alpha^{4} \beta^{7} c_{0} c_{1}{ }^{3}+2664 Q^{2} \alpha^{2} \beta^{9} c_{0} c_{1}{ }^{3}-888 Q^{2} \beta^{11} c_{0} c_{1}{ }^{3}+13440 \alpha^{2} \beta^{8} c_{0}{ }^{3} c_{1}{ }^{4} \\
& -9600 \beta^{10} c_{0}^{3} c_{1}^{4}+43 Q^{4} \alpha^{10} \beta c_{1}-215 Q^{4} \alpha^{8} \beta^{3} c_{1}+430 Q^{4} \alpha^{6} \beta^{5} c_{1}-430 Q^{4} \alpha^{4} \beta^{7} c_{1} \\
& +215 Q^{4} \alpha^{2} \beta^{9} c_{1}-43 Q^{4} \beta^{11} c_{1}+3588 Q^{2} \alpha^{6} \beta^{4} c_{0}{ }^{2} c_{1}{ }^{2}-8660 Q^{2} \alpha^{4} \beta^{6} c_{0}{ }^{2} c_{1}^{2} \\
& +6556 Q^{2} \alpha^{2} \beta^{8} c_{0}{ }^{2} c_{1}{ }^{2}-1484 Q^{2} \beta^{10} c_{0}{ }^{2} c_{1}{ }^{2}+35040 \alpha^{2} \beta^{7} c_{0}{ }^{4} c_{1}{ }^{3}-15840 \beta^{9} c_{0}{ }^{4} c_{1}{ }^{3} \text { ， } \\
& +56 Q^{4} \alpha^{10} c_{0}-232 Q^{4} \alpha^{8} \beta^{2} c_{0}+368 Q^{4} \alpha^{6} \beta^{4} c_{0}-272 Q^{4} \alpha^{4} \beta^{6} c_{0}+88 Q^{4} \alpha^{2} \beta^{8} c_{0} \\
& -8 Q^{4} \beta^{10} c_{0}+4416 Q^{2} \alpha^{6} \beta^{3} c_{0}{ }^{3} c_{1}-8192 Q^{2} \alpha^{4} \beta^{5} c_{0}{ }^{3} c_{1}+4352 Q^{2} \alpha^{2} \beta^{7} c_{0}{ }^{3} c_{1} \\
& -576 Q^{2} \beta^{9} c_{0}{ }^{3} c_{1}+40800 \alpha^{2} \beta^{6} c_{0}{ }^{5} c_{1}{ }^{2}-9120 \beta^{8} c_{0}{ }^{5} c_{1}{ }^{2}+1554 Q^{2} \alpha^{6} \beta^{2} c_{0}{ }^{4} \\
& -2066 Q^{2} \alpha^{4} \beta^{4} c_{0}{ }^{4}+702 Q^{2} \alpha^{2} \beta^{6} c_{0}{ }^{4}-30 Q^{2} \beta^{8} c_{0}{ }^{4}+19200 \alpha^{2} \beta^{5} c_{0}{ }^{6} c_{1}-960 \beta^{7} c_{0}{ }^{6} c_{1} \\
& +1920 \alpha^{2} \beta^{4} c_{0}^{7} \text { ) } \\
& d_{7}=-\frac{1}{7 \beta c_{0}}\left(6 \beta c_{1} d_{6}+5 \beta c_{2} d_{5}+4 \beta c_{3} d_{4}+3 \beta c_{4} d_{3}+2 \beta c_{5} d_{2}+\beta c_{6} d_{1}\right. \text { ， } \\
& \left.+6 c_{0} d_{6}+5 c_{1} d_{5}+4 c_{2} d_{4}+3 c_{3} d_{3}+2 c_{4} d_{2}+c_{5} d_{1}\right) \\
& d_{8}=-\frac{1}{8 \beta c_{0}}\left(7 \beta c_{1} d_{7}+6 \beta c_{2} d_{6}+5 \beta c_{3} d_{5}+4 \beta c_{4} d_{4}+3 \beta c_{5} d_{3}+2 \beta c_{6} d_{2}\right. \text { ， } \\
& \left.+\beta c_{7} d_{1}+7 c_{0} d_{7}+6 c_{1} d_{6}+5 c_{2} d_{5}+4 c_{3} d_{4}+3 c_{4} d_{3}+2 c_{5} d_{2}+c_{6} d_{1}\right) \\
& d_{9}=-\frac{1}{9 \beta c_{0}}\left(8 \beta c_{1} d_{8}+7 \beta c_{2} d_{7}+6 \beta c_{3} d_{6}+5 \beta c_{4} d_{5}+4 \beta c_{5} d_{4}+3 \beta c_{6} d_{3}+2 \beta c_{7} d_{2}\right. \text {, } \\
& \left.+\beta c_{8} d_{1}+8 c_{0} d_{8}+7 c_{1} d_{7}+6 c_{2} d_{6}+5 c_{3} d_{5}+4 c_{4} d_{4}+3 c_{5} d_{3}+2 c_{6} d_{2}+c_{7} d_{1}\right) \\
& d_{10}=-\frac{1}{10 \beta c_{0}}\left(9 \beta c_{1} d_{9}+8 \beta c_{2} d_{8}+7 \beta c_{3} d_{7}+6 \beta c_{4} d_{6}+5 \beta c_{5} d_{5}+4 \beta c_{6} d_{4}\right. \\
& +3 \beta c_{7} d_{3}+2 \beta c_{8} d_{2}+\beta c_{9} d_{1}+9 c_{0} d_{9}+8 c_{1} d_{8}+7 c_{2} d_{7}+6 c_{3} d_{6}+5 c_{4} d_{5} \\
& \left.+4 c_{5} d_{4}+3 c_{6} d_{3}+2 c_{7} d_{2}+c_{8} d_{1}\right) \\
& d_{11}=-\frac{1}{11 \beta c_{0}}\left(10 \beta c_{1} d_{10}+9 \beta c_{2} d_{9}+8 \beta c_{3} d_{8}+7 \beta c_{4} d_{7}+6 \beta c_{5} d_{6}+5 \beta c_{6} d_{5}\right. \\
& +4 \beta c_{7} d_{4}+3 \beta c_{8} d_{3}+2 \beta c_{9} d_{2}+\beta c_{10} d_{1}+10 c_{0} d_{10}+9 c_{1} d_{9}+8 c_{2} d_{8}+7 c_{3} d_{7}{ }^{\prime} \\
& \left.+6 c_{4} d_{6}+5 c_{5} d_{5}+4 c_{6} d_{4}+3 c_{7} d_{3}+2 c_{8} d_{2}+c_{9} d_{1}\right)
\end{aligned}
$$




$$
\begin{aligned}
& d_{12}=-\frac{1}{12 \beta c_{0}}\left(11 \beta c_{1} d_{11}+10 \beta c_{2} d_{10}+9 \beta c_{3} d_{9}+8 \beta c_{4} d_{8}+7 \beta c_{5} d_{7}+6 \beta c_{6} d_{6}\right. \\
& +5 \beta c_{7} d_{5}+4 \beta c_{8} d_{4}+3 \beta c_{9} d_{3}+2 \beta c_{10} d_{2}+\beta c_{11} d_{1}+11 c_{0} d_{11}+10 c_{1} d_{10}+9 c_{2} d_{9}, \\
& \left.+8 c_{3} d_{8}+7 c_{4} d_{7}+6 c_{5} d_{6}+5 c_{6} d_{5}+4 c_{7} d_{4}+3 c_{8} d_{3}+2 c_{9} d_{2}+c_{10} d_{1}\right) \\
& d_{13}=-\frac{1}{13 \beta c_{0}}\left(12 \beta c_{1} d_{12}+11 \beta c_{2} d_{11}+10 \beta c_{3} d_{10}+9 \beta c_{4} d_{9}+8 \beta c_{5} d_{8}\right. \\
& +7 \beta c_{6} d_{7}+6 \beta c_{7} d_{6}+5 \beta c_{8} d_{5}+4 \beta c_{9} d_{4}+3 \beta c_{10} d_{3}+2 \beta c_{11} d_{2}+\beta c_{12} d_{1}+12 c_{0} d_{12}+11 c_{1} d_{11} \\
& \left.+10 c_{2} d_{10}+9 c_{3} d_{9}+8 c_{4} d_{8}+7 c_{5} d_{7}+6 c_{6} d_{6}+5 c_{7} d_{5}+4 c_{8} d_{4}+3 c_{9} d_{3}+2 c_{10} d_{2}+c_{11} d_{1}\right) \\
& d_{14}=-\frac{1}{14 \beta c_{0}}\left(143 \beta c_{1} d_{13}+132 \beta c_{2} d_{12}+11 \beta c_{3} d_{11}+10 \beta c_{4} d_{10}+9 \beta c_{5} d_{9}+8 \beta c_{6} d_{8}+7 \beta c_{7} d_{7}\right. \\
& +6 \beta c_{8} d_{6}+5 \beta c_{9} d_{5}+4 \beta c_{10} d_{4}+3 \beta c_{11} d_{3}+2 \beta c_{12} d_{2}+\beta c_{13} d_{1}+13 c_{0} d_{13}+12 c_{1} d_{12}+11 c_{2} d_{11} \\
& \left.+10 c_{3} d_{10}+9 c_{4} d_{9}+8 c_{5} d_{8}+7 c_{6} d_{7}+6 c_{7} d_{6}+5 c_{8} d_{5}+4 c_{9} d_{4}+3 c_{10} d_{3}+2 c_{11} d_{2}+c_{12} d_{1}\right) \\
& d_{15}=-\frac{1}{15 \beta c_{0}}\left(14 \beta c_{1} d_{14}+13 \beta c_{2} d_{13}+12 \beta c_{3} d_{12}+11 \beta c_{4} d_{11}+10 \beta c_{5} d_{10}+9 \beta c_{6} d_{9}\right. \\
& +8 \beta c_{7} d_{8}+7 \beta c_{8} d_{7}+6 \beta c_{9} d_{6}+5 \beta c_{10} d_{5}+4 \beta c_{11} d_{4}+3 \beta c_{12} d_{3}+2 \beta c_{13} d_{2}+\beta c_{14} d_{1} \\
& +14 c_{0} d_{14}+13 c_{1} d_{13}+12 c_{2} d_{12}+11 c_{3} d_{11} \\
& \left.+10 c_{4} d_{10}+9 c_{5} d_{9}+8 c_{6} d_{8}+7 c_{7} d_{7}+6 c_{8} d_{6}+5 c_{9} d_{5}+4 c_{10} d_{4}+3 c_{11} d_{3}+2 c_{12} d_{2}+c_{13} d_{1}\right)
\end{aligned}
$$

\section{References}

1. Hencky, H. On the stress state in circular plates with vanishing bending stiffness. Z. Angew. Math. Phys. 1915, 63, 311-317. (In German)

2. Föppl, A. Vorlesungen über Technische Mechanik: Bd. Die wichtigsten Lehren der Höheren Elastizitätstheorie; B.G. Teubner: Leipzig, Germany, 1907; Volume 5, pp. 132-144.

3. von Kármán, T. Festigkeitsprobleme im Maschinenbau. In Encyklopedie der Mathematischen Wissenschaften; Klein, F., Müller, C., Eds.; B.G. Teubner: Leipzig, Germany, 1910; pp. 311-385.

4. Timoshenko, S.; Woinowsky-Krieger, S. Theory of Plates and Shells; McGraw-Hill: New York, NY, USA, 1959.

5. Chien, W.Z. Asymptotic behavior of a thin clamped circular plate under uniform normal pressure at very large deflection. Sci. Rep. Natl. Tsinghua Univ. 1948, 5, 193-208. (In Chinese)

6. Alekseev, S.A. Elastic circular membranes under the uniformly distributed loads. Eng. Corpus. 1953, 14, 196-198. (In Russian)

7. Chien, W.Z.; Wang, Z.Z.; Xu, Y.G.; Chen, S.L. The symmetrical deformation of circular membrane under the action of uniformly distributed loads in its portion. Appl. Math. Mech. Engl. Ed. 1981, 2, 653-668.

8. Chien, W.Z.; Chen, S.L. The solution of large deflection problem of thin circular plate by the method of composite expansion. Appl. Math. Mech. Engl. Ed. 1985, 6, 103-118.

9. Arthurs, A.M.; Clegg, J. On the solution of a boundary value problem for the nonlinear Föppl-Hencky equation. Z. Angew. Math. Mech. 1994, 74, 281-284. [CrossRef]

10. Plaut, R.H. Linearly elastic annular and circular membranes under radial, transverse, and torsional loading. Part I: Large unwrinkled axisymmetric deformations. Acta Mech. 2009, 202, 79-99. [CrossRef]

11. Sun, J.Y.; Rong, Y.; He, X.T.; Gao, X.W.; Zheng, Z.L. Power series solution of circular membrane under uniformly distributed loads: Investigation into Hencky transformation. Stuct. Eng. Mech. 2013, 45, 631-641. [CrossRef]

12. Sun, J.Y.; Lian, Y.S.; Li, Y.M.; He, X.T.; Zheng, Z.L. Closed-form solution of elastic circular membrane with initial stress under uniformly-distributed loads: Extended Hencky solution. Z. Angew. Math. Mech. 2015, 95, 1335-1341. [CrossRef]

13. Lian, Y.S.; Sun, J.Y.; Yang, Z.X.; He, X.T.; Zheng, Z.L. Closed-form solution of well-known Hencky problem without small-rotationangle assumption. Z. Angew. Math. Mech. 2016, 96, 1434-1441. [CrossRef]

14. Yang, Z.X.; Sun, J.Y.; Ran, G.M.; He, X.T. A new solution to Föppl-Hencky membrane equation. J. Mech. 2017, 33, N7-N11. [CrossRef]

15. Lian, Y.S.; Sun, J.Y.; Dong, J.; Zheng, Z.L.; Yang, Z.X. Closed-form solution of axisymmetric deformation of prestressed FöpplHencky membrane under constrained deflecting. Stuct. Eng. Mech. 2019, 69, 693-698.

16. Huang, P.F.; Song, Y.P.; Li, Q.; Liu, X.Q.; Feng, Y.Q. A theoretical study of circular orthotropic membrane under concentrated load: The relation of load and deflection. IEEE Access 2020, 8, 126127-126137. [CrossRef] 
17. Lian, Y.S.; Sun, J.Y.; Zhao, Z.H.; Li, S.Z.; Zheng, Z.L. A refined theory for characterizing adhesion of elastic coatings on rigid substrates based on pressurized blister test methods: Closed-form solution and energy release rate. Polymers 2020, $12,1788$. [CrossRef]

18. Li, X.; Sun, J.Y.; Shi, B.B.; Zhao, Z.H.; He, X.T. A theoretical study on an elastic polymer thin film-based capacitive wind-pressure sensor. Polymers 2020, 12, 2133. [CrossRef]

19. Li, X.; Sun, J.Y.; Zhao, Z.H.; He, X.T. Large deflection analysis of axially symmetric deformation of prestressed circular membranes under uniform lateral loads. Symmetry 2020, 12, 1343. [CrossRef]

20. Rao, Y.; Qiao, S.; Dai, Z.; Lu, N. Elastic Wetting: Substrate-Supported Droplets Confined by Soft Elastic Membranes. J. Mech. Phys. Solids 2021, 151, 104399. [CrossRef]

21. Dickey, R.W. On the Transformation of the Föppl Membrane Equations to Ordinary Differential Equations. J. Math. Anal. Appl. 1974, 47, 169-177. [CrossRef]

22. Fichter, W.B. Some Solutions for the Large Deflections of Uniformly Loaded Circular Membranes; NASA: Washington, DC, USA, 1997; p. TP-3658.

23. Campbell, J.D. On the Theory of Initially Tensioned Circular Membranes Subjected to Uniform Pressure. Quart. J. Mech. Appl. Math. 1956, 9, 84-93. [CrossRef]

24. Shi, B.B.; Sun, J.Y.; Huang, T.K.; He, X.T. Closed-form solution for circular membranes under in-plane radial stretching or compressing and out-of-plane gas pressure loading. Mathematics 2021, 9, 1238. [CrossRef]

25. Alekseev, S.A. Elastic annular membranes with a stiff centre under the concentrated force. Eng. Cor. 1951, 10, 71-80. (In Russian)

26. Sun, J.Y.; Hu, J.L.; He, X.T.; Zheng, Z.L. A theoretical study of a clamped punch-loaded blister configuration: The quantitative relation of load and deflection. Int. J. Mech. Sci. 2010, 52, 928-936. [CrossRef]

27. Yang, Z.X.; Sun, J.Y.; Zhao, Z.H.; Li, S.Z.; He, X.T. A closed-form solution of prestressed annular membrane internally-connected with rigid circular plate and transversely-loaded by central shaft. Mathematics 2020, 8, 521. [CrossRef]

28. Lian, Y.S.; Sun, J.Y.; Ge, X.M.; Yang, Z.X.; He, X.T.; Zheng, Z.L. A theoretical study of an improved capacitive pressure sensor: Closed-form solution of uniformly loaded annular membranes. Measurement 2017, 111, 84-92. [CrossRef] 